\title{
Review
}

Martin H.G. Prechtl* and Paul S. Campbell

\section{Metal oxide and bimetallic nanoparticles in ionic liquids: synthesis and application in multiphase catalysis}

\begin{abstract}
Ionic liquids (ILs) are well established as solvents and stabilizing agents for the synthesis of metallic nanoparticles (NPs) in general. The physicochemical properties of ILs and the supramolecular organization in the liquid state are capable of directing the growth of transition metal NPs generated in situ and to subsequently protect and stabilize them. Until now, many different NPs have been successfully synthesized within these media; however, the synthesis of metal oxide and bimetallic alloy or core-shell NPs in ILs is still relatively rare. Herein, we summarize the current state-of-the-art of the synthetic methods for these materials and their application in the broad field of catalysis, including multiphase systems, hydrogenation, dehydrogenation, functionalization, as well as defunctionalization reactions.
\end{abstract}

Keywords: bimetallic; catalysis; ionic liquids; metal oxides; nanoparticles.

*Corresponding author: Martin H.G. Prechtl, Institut für Anorganische Chemie, Universität zu Köln, Greinstr. 6, 50939 Köln, Germany, e-mail: martin.prechtl@uni-koeln.de

Paul S. Campbell: Institut für Anorganische Chemie, Universität zu Köln, Köln, Germany

\section{General introduction}

During the last decade, the research focus on metal nanoparticles (NPs) has significantly spread and numerous publications are continuously published in diverse fields: chemistry, physics, and material science as well as life science and medicine. This reflects the high expectations toward the development of these novel materials. Perhaps the most developed field for the application of metal NPs is catalysis. In catalysis, improvement in efficiency and selectivity of reactions is constantly sought, as well as better recovery and recyclability of the catalytic materials [1-7].

The general definition for NPs is that at least one of their dimensions must fall below $100 \mathrm{~nm}$. However, the most interesting properties related to nanomaterials are best revealed for dimensions below $10 \mathrm{~nm}$. Such particles exhibit high surface-to-volume ratios and unique physicochemical properties located between those of bulk materials and molecular species. The smaller the size, the greater the effect of small changes in size on the properties. In the particular case of catalysis, the catalytic activity of NPs is strongly related to and directed by the energy of the surface atoms, coordination number to neighboring atoms (corner, edge, and face), size, and surrounding ligands and supports $[1,8,9]$. In the particular case of bimetallic (alloy or core-shell) and metal oxide particles, the type and ratio of the atomic composition also plays an important role.

The most common preparation for NP catalysts is the bottom-up concept, treating a metal precursor with a reducing agent. For example, a metal salt or organometallic complex is reduced under $\mathrm{H}_{2}$ pressure, thermal reduction, or treated with a complex hydride such as $\mathrm{NaBH}_{4}$ forming zero-valent metal atoms. These atoms undergo subsequent nucleation forming instable NPs, which must be crucially stabilized to prevent agglomeration toward stable bulk. Common support materials and stabilizers are polymers and oxides, more recently innovative materials such as dendrimers, carbon nanotubes, membranes, ligands, surfactants, and ionic liquids (ILs) [10-12].

Since the last decade, applied catalysis can no longer be imagined without metal NPs. These materials are often described as "semiheterogeneous catalysts," on the frontier of homogeneous and heterogeneous catalysis. To suppress agglomeration of small-sized NPs, it is crucial to add a stabilizing agent, for example, soluble polymers, quartenary ammonium or phosphonium salts, ILs, surfactants, or polyoxoanions providing electronic and/or steric protection $[3,4,10,13]$. It has been revealed that 
ILs, low-temperature molten salts, are among the most important and most investigated classes of stabilizers for bottom-up and more recently also top-down synthetic approaches to metal NPs [14-19].

ILs are defined as salts with melting points below $100^{\circ} \mathrm{C}$. A large number of ILs are even liquid at room temperature. The liquid state is due to the existence of large asymmetric organic cations and the weak coordination of the bulky organic or inorganic anions, influencing the lattice and solvation energies [20]. Some examples of cations and anions relevant for this topic are depicted in Figure 1. ILs present different physicochemical properties in comparison with conventional solvents: negligible vapor pressure, nonflammability, large liquidus range, wide electrochemical window, etc. Therefore, these materials are now found at the forefront of modern chemical research, often named as "green solvents" or "designer solvents." The latter is due to facile adaptability and adjustability, as with a plethora of anion-cation combinations, small and simple alterations are possible to modify the properties of an IL and tailor it to a specific task [21-24]. One specific task is without any doubt their application as reaction media in nanochemistry for the synthesis of NPs and their subsequent application in catalysis. It has been proposed that this is related to their ionic nature and to their three-dimensional organization on the nanoscale. Moreover, it has been shown that ILs may act as stabilizers in a ligand-like coordination mode as surface-ligands to prevent agglomeration and to tune the selectivity of catalyst materials by surface poisoning $[25,26]$. Most recently, it has also been shown that certain ILs are capable of acting themselves as mild reducing agents for a variety of metal complexes and metal salts, in particular the reduction can be induced and controlled by the anion or cation $[13,26,27]$. One of the most investigated IL-class contains imidazolium cations, found by molecular dynamic simulations and coarse-grain modelling to form nanostructures in the liquid state $[28,29]$. This self-organization could entropically drive the formation of nanostructures [30], while ligand-type coordination or ionic double-layers on the metal surface could provide an explanation for the resulting stabilization of the NPs [3]. Furthermore, some of the unique properties exhibited by ILs, in particular negligible vapor pressure, large liquidus range, and high microwave susceptibility, provide opportunities for novel synthesis routes to nanomaterials, as will be discussed herein.

Transition metal NPs for many catalytic transformations in ILs have been reported in several contributions and overview articles [14, 15, 31]. However, only few reports exist on metal oxides and bimetallic alloy or core-shell NPs in ILs, as precursors and/or catalysts for transformations in multiphase systems [13, 32-55]. Their potential applications cover such fields as hydrogenation reactions [32, 33, 36, 40, 45], hydrogenolysis [39, 56], C-C coupling [41], C-C bond cleavage [13], dehalogenation of arenes [55], condensation reactions [54], oxidation [46], as well as electrochemical applications [42, 47, 51-53, 57, 58]. The scope of the present review is to discuss the mentioned traditional catalytic applications, excluding electrochemical applications.

\section{Synthesis and characterization of metal oxide NPs in ILs}

NPs of metal oxides are interesting due to properties deviating from the bulk material, particularly high surface area, and interesting electronic effects. For example, for semiconducting oxides such as $\mathrm{ZnO}$, when scaled down to below their excitonic Bohr radius, quantum confinement is observed, leading to a widening of their band gap.

The typical synthesis routes to such materials involve intensive techniques, such as laser ablation or chemical vapor deposition as well as chemical decomposition
Cations
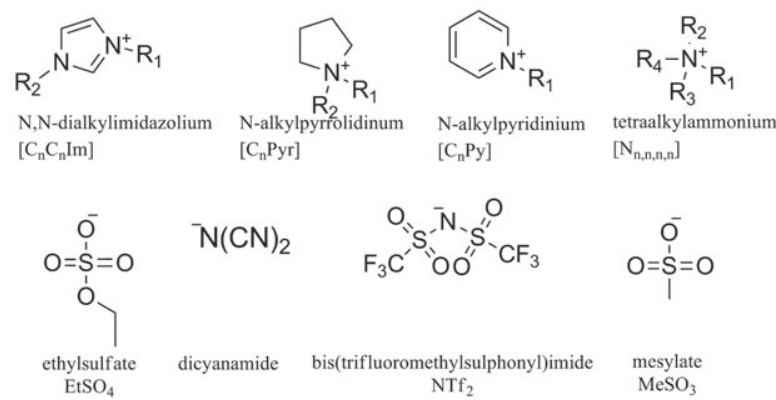

$\mathrm{NTf}_{2}$

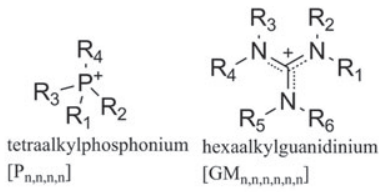

$-\mathrm{BF}_{4} \quad-\mathrm{PF}_{6}$

tetrafluoroborate hexafluorophosphate

Figure 1 Examples of typical cations and anions. 
of precursors under harsh conditions (Scheme 1) [59]. Although typically used for the synthesis of matrices and ceramics, sol-gel techniques can be used to obtain nanoparticular oxides under ambient conditions. However, aqueous sol-gel chemistry typically leads to amorphous precipitates that are transformed into the desired crystalline material by applying a calcination step also necessary to remove surface hydroxyls [60]. The necessity for high-temperature treatment is inconvenient for size- and shape-controlled NP synthesis [61]. Sol-gel processes toward nanoscale oxide materials can be controlled and modulated in purely organic media, where the decomposition of the precursor is not hydrolytic but accompanied by concomitant organic transformations, which have been described previously in detail $[60,62]$. The synthesis of metal oxide NPs is already well reported in traditional aqueous and organic media, and in recent years, numerous reports have appeared focusing on their synthesis in ILs, using their unique ability to template and stabilize nanostructures as well as their facile adaptability. As mentioned previously, the unique physicochemical properties of ILs offer unique opportunities for new synthesis procedures.

Ultrasound-assisted methods have been employed by Mudring et al. in the synthesis of various metal oxide NPs in ILs. Subjecting a medium to ultrasound leads to the rapid creation of growing and imploding bubbles; this leads to localized and short-lived extreme temperatures and pressures, and to high cooling rates, conditions otherwise unattainable and favorable for the formation of wellcrystallized nanostructures. A mixture of copper acetate and sodium hydroxide in the IL $\left[\mathrm{C}_{4} \mathrm{C}_{1} \mathrm{Im}\right]\left[\mathrm{NTf}_{2}\right]$, subjected to $24 \mathrm{~h}$ ultrasound irradiation, led to $\mathrm{CuO}$ nanorods measuring $10 \mathrm{~nm}$ in diameter and 30 to $100 \mathrm{~nm}$ in length [63]. The product could be isolated by washing and centrifugation and its powder X-ray diffraction (XRD) pattern matched closely to that of database monoclinic $\mathrm{CuO}$, confirming the success of the reaction. The size and interesting morphology was observed by transition electron microscopy (TEM). For these semiconductor materials, the band gap could be estimated from ultraviolet-visible (UV-vis) absorption spectra. Here, its value was taken to be the energy at half the absorption of the excitonic peak and found to be $2.36 \mathrm{eV}$. Compared with the bulk gap of

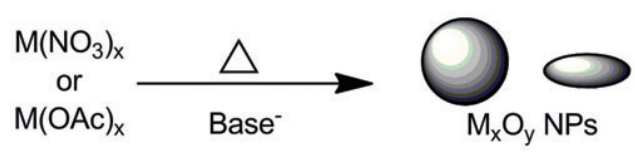

Scheme 1 Base-catalyzed thermal synthesis of metal oxide NPs.
$1.21 \mathrm{eV}$, this is clearly larger, pointing to the quantum electronic effects on this small scale.

Ultrasound-assisted synthesis of titania $\left(\mathrm{TiO}_{2}\right)$ in the IL 1-(3-hydroxypropyl)-3-methylimidazolium bis(trifluoromethylsulfonyl)imide $\left(\left[\mathrm{C}_{3} \mathrm{OHC}_{1} \mathrm{Im}\right]\left[\mathrm{NTf}_{2}\right]\right)$ from titanium tetraisopropoxide led to small spherical nanoparticular $\mathrm{TiO}_{2}$ in the anastase morphology, as confirmed by TEM, X-ray photoelectron spectroscopy (XPS), XRD, and Raman spectroscopy [64]. Again, UV-vis absorption spectra could be used to find the band gap of the material, here calculated equal to $3.29 \mathrm{eV}$, greater than the value for bulk $\mathrm{TiO}_{2}$. Photoluminescence spectra could also be recorded for these samples, exhibiting two principal bands; the first at $383 \mathrm{~nm}$ is due to band-band transitions, whereas a second broader band was attributed to surface trap states. Brunauer-Emmett-Teller techniques could be applied to find the surface area of the as-prepared $\mathrm{TiO}_{2}$ NPs through nitrogen sorption isotherms. The resulting surface area found to be $176.5 \mathrm{~m}^{2} \mathrm{~g}^{-1}$, much higher than commercially available $\mathrm{TiO}_{2}$ but lower than reported $\mathrm{TiO}_{2}$ aerogels.

Zinc oxide is a highly important material, thanks to its direct band gap of $3.37 \mathrm{eV}$ and large exciton binding energy. Similar to the case of $\mathrm{CuO}$, using the ultrasound-assisted technique from zinc acetate hydrate in the $\mathrm{IL}\left[\mathrm{C}_{4} \mathrm{C}_{1} \mathrm{Im}\right]$ [NTf ${ }_{2}$ led to nanorods of $\mathrm{ZnO}$, with diameter approximately $20 \mathrm{~nm}$ and length ranging from 50 to $100 \mathrm{~nm}$ [65]. Electron diffraction and XRD of the isolated material confirmed the formation of the hexagonal wurzite structure of $\mathrm{ZnO}$. The band gap of the as-synthesized material was calculated from the UV-vis spectrum to be $3.31 \mathrm{eV}$, and the material was seen to exhibit strong yellow-green photoluminescence. The luminescence could be due to the recombination of electrons in oxygen vacancy defects with holes in the valence band. ZnO NPs synthesized in such a way have been used as a base for the synthesis of $\mathrm{Ag} / \mathrm{ZnO}$ nanocomposites, where the $\mathrm{ZnO}$ photocatalyzes the reduction of $\mathrm{Ag}^{+}$in daylight and acts as a support for the smaller NPs formed. The resulting materials can thereafter be applied as enhanced photocatalysts [63].

Using ultrasound synthesis method in the preparation of $\mathrm{ZnO}$ nanomaterials from zinc acetate in the $\mathrm{IL}\left[\mathrm{C}_{3} \mathrm{OHC} \mathrm{OH}_{1} \mathrm{Im}\right]$ $\left[\mathrm{BF}_{4}\right]$, Hou et al. prepared dendritic materials on the submicrometer scale. XRD patterns matched closely database $\mathrm{ZnO}$, and photoluminescence spectra showed typical bands for band-gap emission and oxygen vacancy defects. It is worth noting that, in this procedure, the reaction mixture consisted of equal volumetric amounts of deionized water and the IL. The proposed mechanism for this dendrimer formation involves the adsorption of IL cation on the $\mathrm{ZnO}$ nuclides, leading to anisotropic growth [66]. 
Alammar and Mudring also recently reported the importance of the nature of the IL used and the exact reaction conditions on the resulting particle size and morphology of $\mathrm{ZnO}$ nanomaterials [67]. Using their established ultrasound synthesis in neat ILs, several parameters were varied, each time ensuring that other reaction variables were kept constant. First, it was seen that the increasing cation alkyl chain length to $\mathrm{C}_{8}$ and $\mathrm{C}_{10}$ leads to the formation of nanospheres instead of nanorods. This was explained by considering the added steric bulk in the case of the longer chains, inhibiting close interaction with the $\mathrm{ZnO}$ surface and thus limiting the directional growth necessary for the formation of nanorods. The nature of the interaction of the IL cation with the surface was probed by blocking the $\mathrm{C}_{2}-\mathrm{H}$ position of the ring using 1-ethyl-2,3-dimethylimidazolium or eliminating the $\pi$-system using trimethylbutylammonium. Use of this IL also led to similarly sized nanorods, meaning that the principal interaction is probably neither through the $\mathrm{C}_{2}-\mathrm{H}$ position nor through $\pi$-interactions. The anion effect was also probed by replacing $\mathrm{NTf}_{2}$ with the more strongly coordinating $\mathrm{SCN}$, also leading to similarly sized nanorods. It was concluded that the cation has the most important effect on the resultant particle size and morphology. With respect to reaction conditions, shorter reaction times produced nanosheets, which converted to the usual nanorods with time, while increasing $\mathrm{NaOH}$ concentration favored the formation of nanospheres. The same reaction carried out by traditional heating (ionothermal) methods, whereby the reactant mixture in IL was kept at $170^{\circ} \mathrm{C}$ for $20 \mathrm{~h}$, led to rod-like nanostructures, although this time with definite prismatic tips. This could be explained by considering the relative free energy of each exposed crystal phase, coupled with the favorable conditions for good crystallization when using higher temperatures and longer reaction times. Sample scanning electron micrographs (SEM) from the study are given in Figure 2, nicely illustrating the different morphologies possible, also summarized in Table 1 , entry 6.

It should be noted that ultrasound-assisted synthesis of metal oxide NPs is not unique to IL media. For example, precipitation of $\mathrm{ZnO}$ platelets on the micrometer scale was achieved under ultrasound irradiation of an aqueous solution of $\mathrm{ZnCl}_{2}$ and $\mathrm{KOH}[76]$. Addition of a capping agent, such as cetyltrimethylammonium bromide (CTAB), can bring the size of the NPs down to the nanoscale in a controlled manner [77]. Particularly as CTAB resembles an IL in its molecular structure, this example emphasizes the major advantage of ILs in nanosynthesis: the ability to act simultaneously as solvent and capping agent while also providing some control over the size and morphology of the resulting materials.

A remarkably simple method was recently reported for the synthesis of uniformly sized ZnO NPs by simply grinding the precursors and reagents $\left(\mathrm{Zn}(\mathrm{OAc})_{2}\right.$ and $\left.\mathrm{NaOH}\right)$ in the presence of solid $\left[\mathrm{C}_{4} \mathrm{C}_{1} \mathrm{Im}\right][\mathrm{Cl}][70]$. This rapid method
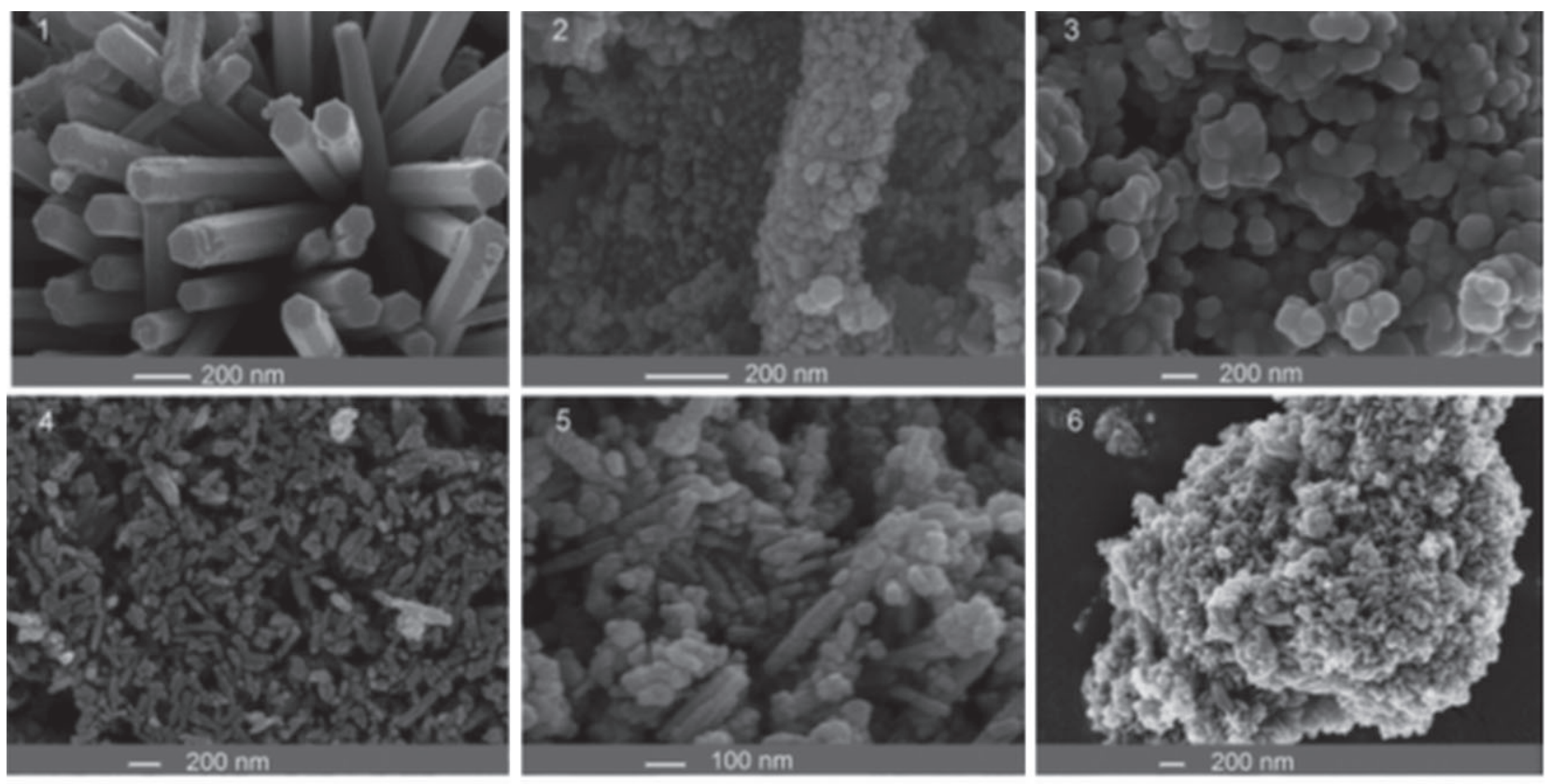

Figure 2 SEM images of $Z n O$ nanostructures generated by ultrasound-assisted decomposition of $Z n(O A c){ }_{2}$ in (1) $\left[C_{4} C_{1} \operatorname{Im}\right]\left[\mathrm{NTf}_{2}\right],(2)\left[C_{8} C_{1} \operatorname{Im}\right]$

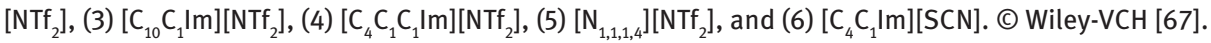




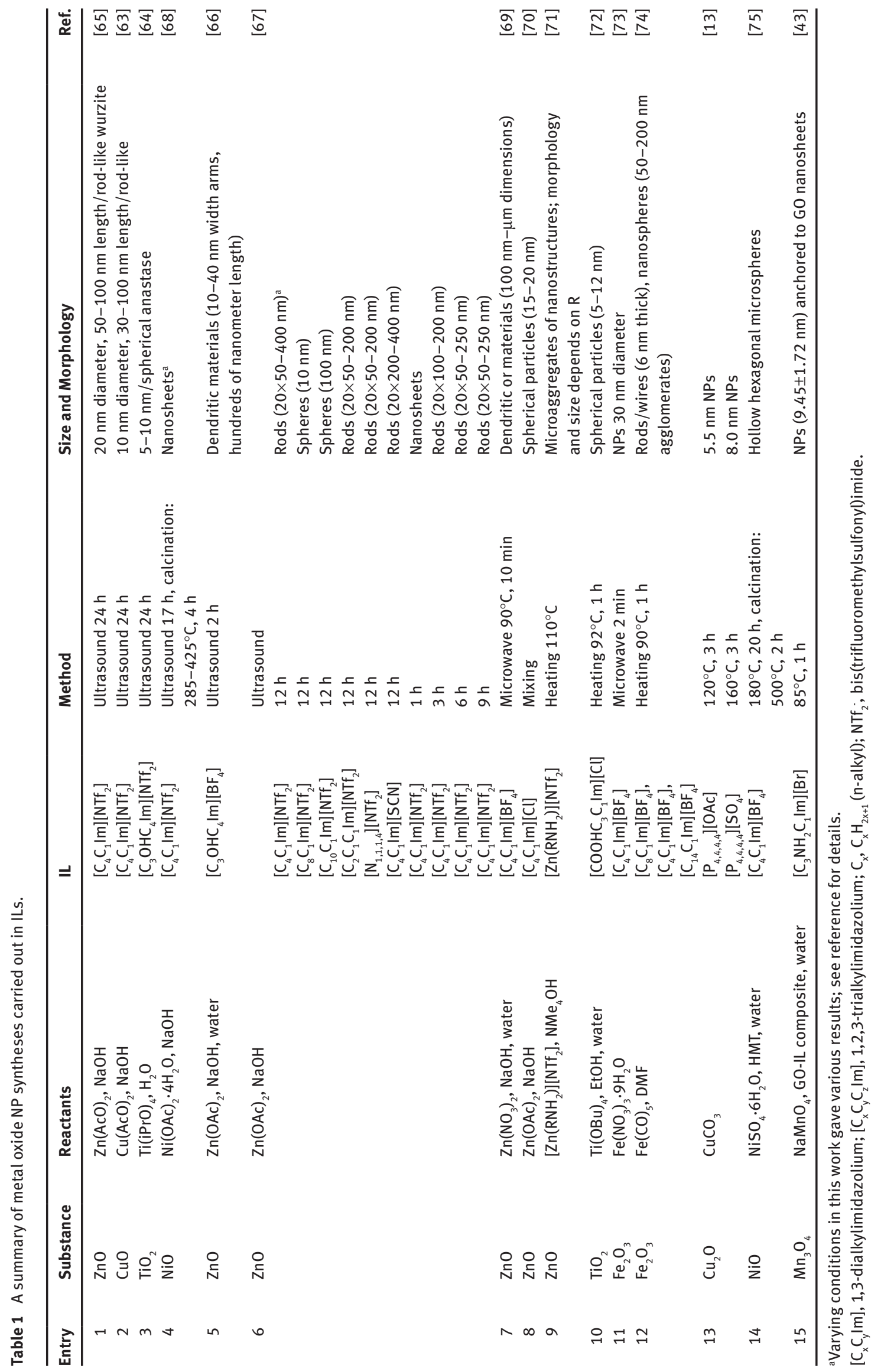


involved no complicated equipment and NPs could be isolated by simply washing the resultant white batter with water and ethanol. TEM and SEM images showed the presence of spherical particles sized from 15 to $20 \mathrm{~nm}$. From the line broadening of XRD patterns, the Debye-Scherrer formula could be applied (Eq. 1) to find $\tau$, the average crystallite size, taking $\beta$ as the line broadening and $K$ as the shape factor ( $K=0.89$ for spherical particles). Herein, this was found equal to $15 \mathrm{~nm}$, in good agreement with microscopy results.

$$
\tau \approx \frac{K \lambda}{\beta \cos \theta},
$$

Another interesting wide band-gap material synthesized on the nanoscale using sonochemical methods in ILs is NiO. As well as in catalysis, this material has found use in gas sensors, magnetic materials, fuel cell electrodes, electrochromic films, and many more. Here, treatment of the precursors under ultrasound irradiation led to $\mathrm{Ni}(\mathrm{OH})_{2}$ nanostructures. These were converted to $\mathrm{NiO}$ by calcination at high temperatures $\left(285-400^{\circ} \mathrm{C}\right)$. The conversion to cubic NiO was also observed by powder XRD measurements. SEM showed that $\mathrm{Ni}(\mathrm{OH})_{2}$ and $\mathrm{NiO}$ were both formed as nanosheets, with dimensions approximately 200 to $300 \times 300 \mathrm{~nm}$ and sheet thickness of $40 \mathrm{~nm}$ for $\mathrm{Ni}(\mathrm{OH})_{2}$ and $55 \mathrm{~nm}$ for NiO. The band gap calculated from UV-vis absorption measurements was found to be $3.3 \mathrm{eV}$, lower than the bulk value. This unexpected red shift of absorption was explained by considering the potentially high number of defects and vacancies in the as-prepared samples. The magnetic behavior of the as-prepared nanomaterial was investigated by room temperature magnetic measurements; interestingly, the resulting hysteresis curve was typical for ferromagnetic behavior despite the fact that bulk NiO is known to be antiferromagnetic. This was attributed to the high shape anisotropy of the material. The as-prepared $\mathrm{NiO}$ nanomaterials were also shown to exhibit an excellent specific capacitance as well as high Brunauer-Emmett-Teller surface area and pore size dependent on the calcination temperature. Similar to $\mathrm{ZnO}$ nanomaterials synthesized sonochemically in ILs, the nature of the IL used as well as the experimental conditions were found to influence the nanostructure morpho$\log [68]$.

Traditional heating methods for the production of metal oxides can be easily transposed to ILs. So-called "ionothermal" synthesis is analogous to "hydrothermal" and "solvothermal" synthesis, the major advantage being that ILs exhibit a much wider liquidus range and of course may be used without stabilizing additives for the resultant nanomaterials. This means that high temperatures are possible without the need for high-pressure equipment, where ILs are used pure. NiO materials have also been synthesized using ILs via ionothermal synthesis. Nickel sulfate was reduced in mixtures of water and the IL $\left[\mathrm{C}_{8} \mathrm{C}_{1} \mathrm{Im}\right]\left[\mathrm{BF}_{4}\right]$ using hexamethylenetetramine (HMT) as the base, and the mixture was heated to $180^{\circ} \mathrm{C}$ in an autoclave for $20 \mathrm{~h}$ [75]. $\mathrm{Ni}(\mathrm{OH})_{2}$ nanostructures also resulted from this synthesis method, which had to be calcined at $500^{\circ} \mathrm{C}$ to obtain the desired $\mathrm{NiO}$ material. This was isolated as hollow hexagonal microspheres with a high specific surface area. From observation of the micrographs, these microspheres appear to be made up of nanosized fragments of NiO. Their exact morphology was found to vary with IL concentration used. It was postulated that due to the high viscosity but low interfacial tension in ILs, nanoseeds appear quickly but diffuse slowly, leading to the microsphere aggregates.

$\mathrm{TiO}_{2}$ NPs were also prepared using a carboxylatebearing IL as an additive in ethanol and hydrolyzing the precursor $\mathrm{Ti}(\mathrm{OBu})_{4}$ under reflux for $1 \mathrm{~h}$. The washed, centrifuged, and dried product was described as an ILfunctionalized nanomaterial. XRD proved the presence of pure anatase-phase $\mathrm{TiO}_{2}$ and SEM and TEM showed the presence of small NPs. These were subsequently used as a support for gold nanocatalysts [72].

Iron oxide $\left(\mathrm{Fe}_{2} \mathrm{O}_{3}\right)$ nanomaterials were synthesized in mixtures of $\left[\mathrm{C}_{8} \mathrm{C}_{1} \mathrm{Im}\right]\left[\mathrm{BF}_{4}\right]$ and $N, N$-dimethylformamide (DMF) by simple ionothermal decomposition of $\mathrm{Fe}(\mathrm{CO})_{5}$, heating to $90^{\circ} \mathrm{C}$ for $1 \mathrm{~h}$ [74]. The resulting morphology of the nanomaterials could be varied from short nanorods (30 nm long and $6 \mathrm{~nm}$ wide) to long nanowires. Changing the IL also led to a change in morphology, with spherical structures observed in the case of $\left[\mathrm{C}_{4} \mathrm{C}_{1} \mathrm{Im}\right]\left[\mathrm{BF}_{4}\right]$ and $\left[\mathrm{C}_{14} \mathrm{C}_{1} \mathrm{Im}\right]\left[\mathrm{BF}_{4}\right]$. The particles produced could be used in medical applications due to their magnetic properties.

Due to their ionic nature and high polarizability, ILs are well known to be good microwave susceptors. This leads to the unique opportunity of extremely high heating rates, ideal for the rapid formation of nuclides needed in controlled nanosynthesis. For instance, a domestic microwave was used by Jacob et al. to produce $\mathrm{Fe}_{2} \mathrm{O}_{3} \mathrm{NPs}$ in ILs [73]. In the IL $\left[\mathrm{C}_{4} \mathrm{C}_{1} \mathrm{Im}\right]\left[\mathrm{BF}_{4}\right], \mathrm{Fe}\left(\mathrm{NO}_{3}\right)_{3} 9 \mathrm{H}_{2} \mathrm{O}$ was irradiated in a $900 \mathrm{~W}$ microwave for $2 \mathrm{~min}$, resulting in $\mathrm{Fe}_{2} \mathrm{O}_{3} \mathrm{NPs}$, as confirmed by TEM and XRD. However, increasing the reaction time to 10 min leads to the formation of fluoride NPs due to the decomposition of the $\mathrm{BF}_{4}$ anion, a fact that has since been developed in other groups for the generation of fluoride nanomaterials in ILs $[78,79]$.

Microwave irradiation was also used to synthesize $\mathrm{ZnO}$ nanostructures from $\mathrm{Zn}\left(\mathrm{NO}_{3}\right)_{2}$ and $\mathrm{NaOH}$ in water in the presence of the IL $\left[\mathrm{C}_{4} \mathrm{C}_{1} \operatorname{Im}\right]\left[\mathrm{BF}_{4}\right]$ [69]. Because the 
quantity of water and IL was varied, different morphologies of $\mathrm{ZnO}$ nanostructures were formed. When the medium was majoritarily IL, ZnO needles grew, whereas, with higher amounts of water, dendritic flower-type structures were observed. The latter observation is remarkably similar to that observed in Hou et al.'s work, where a mixture of water and IL was also used to synthesize $\mathrm{ZnO}$ under ultrasound irradiation [66].

Small $\mathrm{Cu}_{2} \mathrm{O}$ NPs were recently formed by thermal treatment of $\mathrm{CuCO}_{3}$ in various ILs, where the IL was used as a reactant to reduce the precursor in situ [13]. Various ILs were tested in this respect; however, it was found that the acetate anion was necessary for an efficient reduction of the precursor, as confirmed by nuclear magnetic resonance and mass spectrometry studies, whereas phosphonium cations ILs helped stabilize the small spherical NPs. In contrast, when phosphonium sulfate was used, the reduction process occurs differently, induced by the cation via a Hofmann elimination. The presence of $\mathrm{Cu}_{2} \mathrm{O}$ was confirmed by XRD measurements, whereas the presence of small NPs ( $5.0 \mathrm{~nm}$ ) was confirmed by TEM.

As mentioned previously, ILs are highly adaptable and can be designed to accomplish a specific task. As such, an IL precursor has been reported by Zhu et al. for the synthesis of $\mathrm{ZnO}$ nanostructured materials [71]. The zinc-containing cation shown in Figure 3 could be decomposed by the addition of a methanolic solution of tetramethylammonium hydroxide to the pure IL and heating to $110^{\circ} \mathrm{C}$ for several hours. The size and morphology of the resultant NPs varied with the size of the amine ligand. Consequently, the amine ligand is thought to act as a capping agent for the resultant NPs. Reaction temperature and hydroxide concentration were also found to affect resulting particle morphologies.

$\mathrm{Mn}_{2} \mathrm{O}_{4}$ NPs were also produced anchored to graphene oxide (GO) nanosheets with the aid of the IL 1-(3-aminopropyl)-3-methylbromide. The precursor $\mathrm{NaMnO}_{4}$ was reduced in the presence of the IL-functionalized GO, producing small crystalline NPs with high size control. The resulting hybrid material was proven to be an efficient electrocatalyst [43].

\section{Synthesis and characterization of bimetallic NPs in ILs}

The synthesis of zero-valent metallic NPs in IL is already a well-established field, with many different metals having been studied, as recently comprehensively reviewed [14, $15,80]$. Generally, this occurs via the decomposition or reduction of a metal salt or organometallic complex in situ in an IL, where the IL acts as a solvent and stabilizer for the resultant NPs.

Many reports exist for the synthesis of bimetallic NPs in traditional solvents, but interestingly relatively few have attempted this in ILs. Thus far, the synthesis of bimetallic NPs in ILs has been undertaken in a similar way to the synthesis of monometallic NPs in ILs, only combining two different metal precursors in the synthesis. The major challenge is to determine the nature of the resultant materials. When embarking upon bimetallic NP synthesis, possible results include alloys (i.e., homogeneous crystalline mixture of both metals), fused clusters,
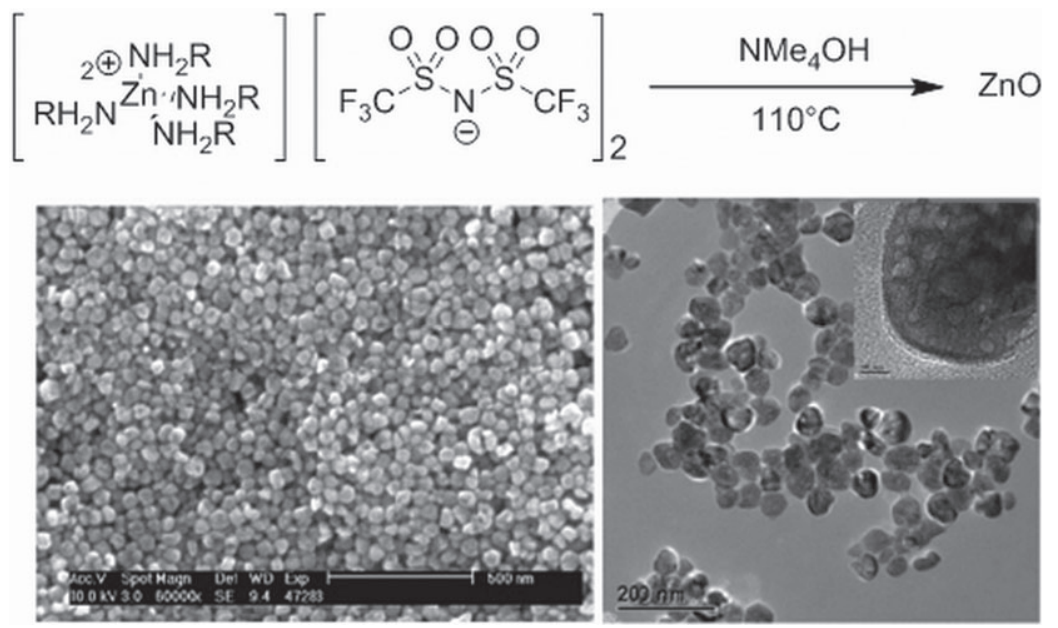

Figure 3 IL precursor for ZnO NP synthesis.

Bottom, SEM (left) and TEM (right) images of ZnO NPs resulting when R=octyl. @ ACS. Adapted with permission from [71]. 
core-shell, or simply mixtures of monometallic NPs, as depicted in Scheme 2. The formation of an alloy can be predicted by consulting the phase diagram of the two metals, paying attention to their miscibility [81]. However, in NP synthesis, many more factors come into play. For instance, different reaction kinetics and surface free energies of the two metals mean that pure alloy formation is never guaranteed. When an immiscible mixture of metals is used, alloy formation is unlikely and the formation of core-shell, fused cluster, or monometallic NPs depends on the reaction kinetics and surface interactions with the surrounding medium. It is clear to see that each of the possible products presents a different surface; thus, in catalysis, it is important to know which species has been formed.

Janiak et al. previously reported the generation of metallic NPs via the thermal/photo-decomposition of metal carbonyl complexes in ILs [82-85]. Andanson et al. found that this could be applied to the synthesis of bimetallic NPs (e.g., Fe-Ru NPs) by the decomposition of a mixture of $\mathrm{Fe}_{2}(\mathrm{CO})_{9}$ and $\mathrm{Ru}_{3}(\mathrm{CO})_{12}$ in the IL $\left[\mathrm{C}_{4} \mathrm{C}_{1} \mathrm{Im}\right]\left[\mathrm{BF}_{4}\right]$ or $\left[\mathrm{C}_{4} \mathrm{C}_{1} \mathrm{Im}\right]$ [PF 6 [ [45]. Different atomic ratios of Fe and Re were used, namely, 9:1, 3:1, and 1:1, with only the latter expected to form alloys according to the phase diagram. For a 1:1 ratio, it was deduced that bimetallic NPs were formed as smaller sizes with narrower size distribution $(1.65 \pm 0.30 \mathrm{~nm})$ were produced than when using $\mathrm{Ru}$ alone $(2.91 \pm 0.75 \mathrm{~nm})$ under the same conditions. It was also deduced from the catalytic behavior that iron enrichment of the NP surface did not occur. In contrast, a solvothermal method in EG, requiring a polyvinylpyrrolidone (PVP) stabilizer, led to larger Fe-Ru NPs with poorer size control [86].

The hydrogen-induced reduction and decomposition of organometallic precursors is a widely reported route to metallic NPs in ILs. Santini's group used this method in an attempt to generate bimetallic $\mathrm{Ru}-\mathrm{Cu} \mathrm{NPs}$. The precursors mesityl copper(I) (CuMes) and ( $\eta^{4}-1,5$-cyclooctadiene) ( $\eta^{4}-1,3,5$-cyclooctatriene)ruthenium $(\mathrm{Ru}(\mathrm{COD})(\mathrm{COT}))$ were decomposed simultaneously in the $\mathrm{IL}_{2}\left[\mathrm{C}_{4} \mathrm{C}_{1} \mathrm{Im}\right]\left[\mathrm{NTf}_{2}\right]$ under 9 bar $\mathrm{H}_{2}$ at $100^{\circ} \mathrm{C}$ for $4 \mathrm{~h}$, resulting in small spherical NPs with a narrow size distribution $(2.5 \pm 0.6 \mathrm{~nm})$. Because, under the same conditions, both $\mathrm{Cu}$ and $\mathrm{Ru}$ alone gave larger NPs, it was inferred that the resulting NPs were indeed bimetallic. Further evidence was provided by energy-dispersive X-ray spectroscopy (EDX), where both $\mathrm{Cu}$ and $\mathrm{Ru}$ were identified; however, high-resolution TEM (HR-TEM) showed crystalline cores matching closely to metallic hcp ruthenium. It was therefore proposed that $\mathrm{Ru}-\mathrm{Cu}$ core-shell NPs resulted [87]. Indeed, when varying the molar ratio of the precursors used, the resulting NP sizes corresponded to the expected size of Ru-Cu NPs with a $2 \mathrm{~nm}$ Ru core and a closely packed Cu shell, as illustrated in Figure 4. This was attributed to the different kinetics of the decomposition reactions; $\mathrm{Ru}(\mathrm{COD})(\mathrm{COT})$ decomposes more rapidly than CuMes. Decomposition of the latter occurs preferentially on the surface of ready-formed $\mathrm{Ru}$ nuclides. This hypothesis was further supported by deuterium labeling experiments, whereby Ru NPs formed under $\mathrm{D}_{2}$ were seen to catalyze the reduction of CuMes under Ar, with the deuterated mesitylene by-product being detected by mass spectrometry. The deuterium originated from the $\mathrm{Ru}$ NP surface, which is known to be covered in hydrogen after formation of NPs [19].

The synthesis of Ru-Cu NPs in IL was also attempted via a combined physical and chemical approach [19]. Ru NPs were first synthesized by decomposition of $\mathrm{Ru}(\mathrm{COD})$ (COT); subsequently, metallic copper was evaporated into the medium by physical vapor deposition (PVD). In this case, mixtures of monometallic NPs were formed.

Bimetallic Ru-Cu NP catalysts have also been anchored to bentonite (BEN; clay) particles using the IL 1,1,3,3-tetramethylguanadinium lactate through the reduction of the

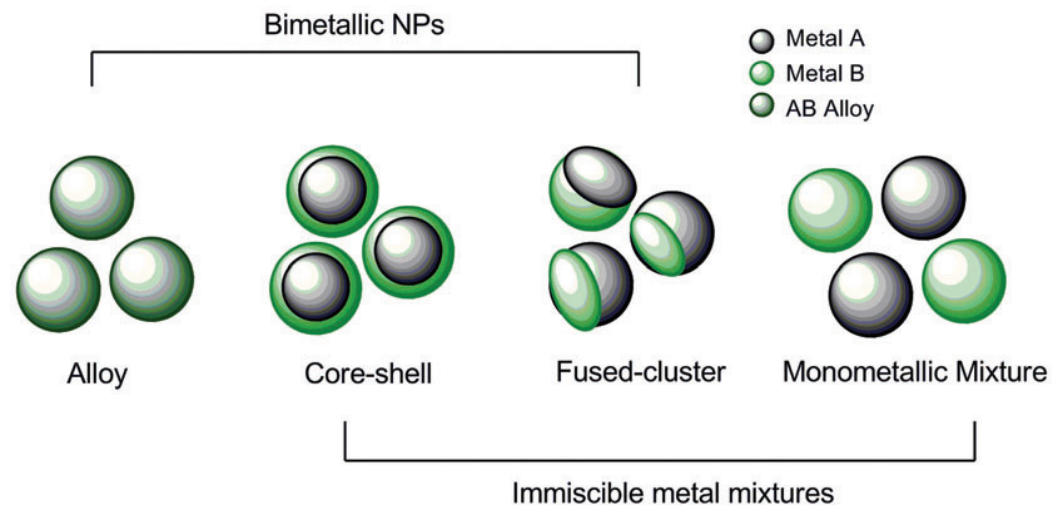

Scheme 2 Possible results from bimetallic NP synthesis. 


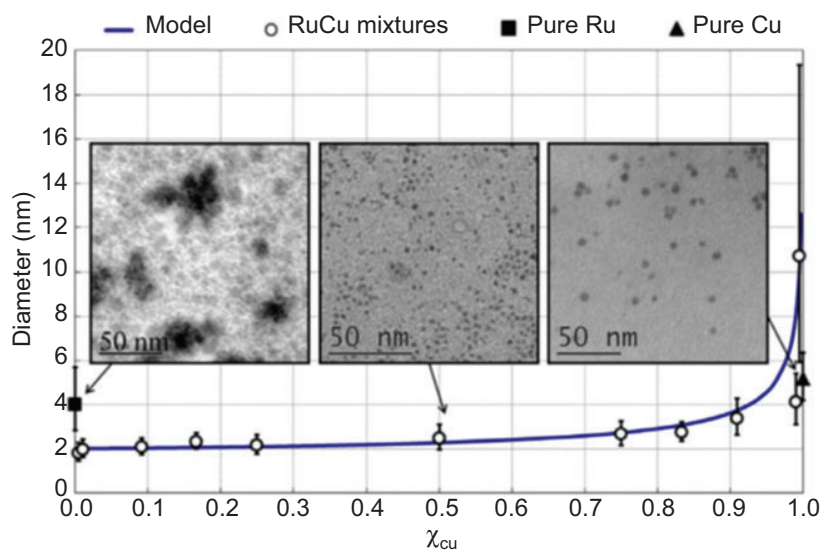

Figure 4 Size evolution of Ru-Cu bimetallic NPs with initial composition of the solution.

The size of Ru-Cu NPs (white circles) is plotted as a function of the molar fraction of $\mathrm{Cu}\left(\chi_{\mathrm{cu}}\right)$ in solution and compared with pure $\mathrm{Ru}$ and pure $\mathrm{Cu}$. Error bars correspond to \pm 2 standard deviations in the log-normal distribution. The solid line represents the size evolution expected from the formation of core-shell NPs with a $2 \mathrm{~nm}$ core of $\mathrm{Ru}$ and a shell of closely packed Cu. TEL images of $\chi_{\text {cu }}$ values $0,0.5$, and 1 are also shown. (c) Elsevier [19].

precursors $\mathrm{RuCl}_{3} \cdot 3 \mathrm{H}_{2} \mathrm{O}$ and $\mathrm{Cu}\left(\mathrm{NO}_{3}\right)_{2} \cdot 3 \mathrm{H}_{2} \mathrm{O}$ in situ. Binding energies of $\mathrm{Ru}$ and $\mathrm{Cu}$ were found by XPS to be shifted in the bimetallic materials compared with the monometallic equivalents, suggesting a close interaction between the two metals [39].

Santini's group has also reported the synthesis of bimetallic Ru-Ta NPs using Ru(COD)(COT) and Tris(neopentyl) neopentylidene tantalum $(\mathrm{V})\left(\mathrm{TaNp}_{3}=\mathrm{Np}\right)$ as precursors [88]. Similarly, it was found that mixtures of metals gave smaller NPs $(2.5 \mathrm{~nm})$ under the same conditions than either of the metals alone, strongly indicating the formation of bimetallic NPs. The nature of the resulting NPs is more difficult to determine. Unlike $\mathrm{Ru}-\mathrm{Cu}, \mathrm{Ru}$ and $\mathrm{Ta}$ are highly miscible. HR-TEM of the NPs formed could confirm either the crystal structure of $\mathrm{Ru}$ or $\mathrm{Ru}_{4} \mathrm{Ta}$, as the structure of both is highly similar. It is important to note that such small zero-valent Ta or Ta-containing NPs have never produced through mild chemical routes in other media.

Synthesis of bimetallic NPs of noble metal mixtures such as Pt-Au has been widely studied in various media and usually requires deposition onto a substrate or chemical reduction of a precursor in the presence of capping agents such as PVP [89]. Scott et al. have focused on the preparation of Pd-Au bimetallic NPs in ILs, both directly in ILs and by transfer from another solvent after synthesis. For example, the preparation of Pd-Au NPs (3:1) was carried out both directly in the IL $\left[\mathrm{C}_{4} \mathrm{C}_{1} \mathrm{Im}\right]\left[\mathrm{PF}_{6}\right]$ and in methanol with a PVP stabilizer followed by transfer into this IL. These were prepared by the simultaneous reduction of $\mathrm{HAuCl}_{4}$ and $\mathrm{K}_{2} \mathrm{PdCl}_{4}$ using $\mathrm{NaBH}_{4}$. Alongside PVP, 1-methylimidazole and 1-(3-aminopropyl)-3-methylimidazolium hexafluorophosphate additives were tested as additional stabilization agents. The use of these led to smaller NPs than the IL alone while also limiting the aggregation of the NPs over time [36, 38]. Pd-Au NPs were also prepared by the same group in a sequential growth process in a mixture of the IL trihexyl(tetradecyl)phosphonium chloride $\left[\mathrm{P}_{6,6,6,14}\right][\mathrm{Cl}]$ in dioxane. First, Au NP seeds were produced via the reduction of $\mathrm{HAuCl}_{4}$ with $\mathrm{LiBH}_{4}$ followed by the addition of ascorbic acid and $\mathrm{K}_{2} \mathrm{PdCl}_{4}$ in methanol. The resulting NPs were small with a narrow size distribution $(3.7 \pm 1.0 \mathrm{~nm})$ as evidenced by TEM. The characterization of the NPs was achieved by extended X-ray absorption fine structure, whereby a shift in periodicity on the $\mathrm{Au} k$-space, with respect to pure Au NPs, suggested some degree of alloying in the NP. Furthermore, in the Pd data, Pd-Pd and Pd-Au coordination environments dominated over minimal $\mathrm{Pd}-\mathrm{O}$ or $\mathrm{Pd}-\mathrm{Cl}$ contributions [55].

Synthesis of Pd-Au NPs in an IL designed to partake in the reduction of the metal precursors, namely, 1-(2-hydroxyethyl)methylimidazolium bis(trifluoromethylsulfonyl) imide $\left[\mathrm{C}_{2} \mathrm{OHC}_{1} \mathrm{Im}\right]\left[\mathrm{NTf}_{2}\right]$, was proposed by Yuan et al. [55]. In this IL, mixtures of $\mathrm{Pd}(\mathrm{OAc})_{2}$ and $\mathrm{Au}(\mathrm{OAc})_{3}$ can be reduced by thermal treatment $\left(120^{\circ} \mathrm{C}\right.$ for $\left.120 \mathrm{~min}\right)$ where the acetate anion acts as reducing agent $[13,55$, 90]. The resultant NPs were characterized by TEM and HR-TEM, showing small and highly crystalline materials crystallized with an fcc structure. TEM-EDX showed that the composition of the materials corresponded closely to the original ratio of precursors used, whereas XRD patterns varied according to Vegard's law with varying composition, whereby the lattice parameters of an alloy vary with the concentration of its substituents. Nonetheless, the higher than expected Pd binding energy intensities observed by XPS suggested a surface enrichment of $\mathrm{Pd}$ in the alloy NPs, thus a partial core-shell structure. $\mathrm{X}$-ray absorption spectroscopy corroborated this finding, leading to the speculation of slower reduction of $\mathrm{Pd}(\mathrm{OAc})_{2}$ than Au causing the Pd enrichment of the shell, similar to the results proposed by Santini's group for core-shell Ru-Cu NPs.

Zhang et al. overcame the faster reduction of Pd to synthesize Pd-Au core-shell NPs in an IL/water emulsion. $\left(\mathrm{NH}_{4}\right)_{2} \mathrm{PdCl}_{6}$ dissolved in the aqueous phase was reduced at $45^{\circ} \mathrm{C}$ by the hydroxyl group of the emulsifying agent Triton X-100. Subsequently, $\mathrm{HAuCl}_{4}$ dissolved in $\left[\mathrm{C}_{4} \mathrm{C}_{1} \mathrm{Im}\right]$ $\left[\mathrm{PF}_{6}\right]$ was reduced on the surface of the Pd cores, provoking the temporary release of $\mathrm{Pd}^{2+}$, which was subsequently reduced yet again on the surface of the NPs [46]. 
An inorganic molten salt consisting of a mixture of $\mathrm{LiNO}_{3}$ and $\mathrm{KNO}_{3}$ was used as a medium for the generation of Pt-Cu alloy NPs via the simultaneous thermal reduction of $\mathrm{Pt}\left(\mathrm{NH}_{3}\right)_{2} \mathrm{C}_{2} \mathrm{O}_{4}$ and $\mathrm{Cu}\left(\mathrm{NO}_{3}\right) \cdot 3 \mathrm{H}_{2} \mathrm{O}$. This method presented a "green" aspect by avoiding the use of volatile organic materials and resulted in concave nanostructures of Pt-Cu alloy, as shown by SEM, TEM, EDX, and XRD [51].

\subsection{Physical "top-down" synthesis methods}

Thanks to their generally very low vapor pressure, physical "top-down" methods may also be used for the synthesis of NPs in ILs. Sputter deposition is a PVD technique, whereby a bulk substrate is bombarded with $\mathrm{Ar}^{+}$ions, provoking the physical ejection of atoms or clusters from the surface. The sputtered material can subsequently form thin films. However, if the sputtering is performed onto ILs, small NPs may be formed instead of thin films. Here, the negligible vapor pressure of ILs is used, as ultrahigh vacuum conditions can be applied to these solvents without fear of evaporation. This technique was first successfully implemented by Torimoto's group, who produced Au-Ag NPs by simultaneous sputtering of the two metals onto the IL $\left[\mathrm{C}_{4} \mathrm{C}_{1} \mathrm{Im}\right]\left[\mathrm{PF}_{6}\right]$ [91]. Several tests involving different $\mathrm{Au} / \mathrm{Ag}$ ratios were performed and the authors were able to prove, through both UV-vis measurements of the varying surface plasmon resonance and EDX of the highly crystalline NPs, that Ag-Au alloy NPs were indeed formed. The same group have also reported the production of small $(<5 \mathrm{~nm}) \mathrm{Au}$-Pt NPs via the same technique [49]. By systematically changing the $\mathrm{Au} / \mathrm{Pt}$ ratio on the targets, they were able to prove by $\mathrm{X}$-ray fluorescence elemental analysis, a direct correlation with the ratio of the metals in the resultant materials, with a factor of 1.3. The greater quantity of $\mathrm{Au}$ in the latter was explained in terms of differing sputtering rates of the two metals. Similar to $\mathrm{Au}$ and $\mathrm{Ag}, \mathrm{Au}$ and $\mathrm{Pt}$ are highly miscible metals; thus, in each case, alloy NPs were formed. XRD results presented an fcc structure corresponding to $\mathrm{Au}$ and Pt. It was seen that, as the Au amount increased, the $d$-spacings were reduced linearly, explained in terms of Vegard's law.

The sputtering of early transition metals, namely, $\mathrm{W}, \mathrm{Mo}, \mathrm{Nb}$, and $\mathrm{Ti}$, into the $\mathrm{IL}\left[\mathrm{C}_{4} \mathrm{C}_{1} \mathrm{Im}\right]\left[\mathrm{BF}_{4}\right]$ resulted in the formation of small anisotropic NPs. Closer investigation by XPS revealed that these NPs consisted mostly of oxidic phases, although it is unclear how this oxidation occurred, possibly by residual water or by oxygen impurities in the argon gas flow [92].

Mudring's group have also reported PVD as a path to IL NP suspensions. Under ultrahigh vacuum conditions, allowed due to the low vapor pressure of ILs, a desired material is evaporated into a film of IL using resistive heating or an electron gun, resulting in small, well-dispersed NPs. This method has been used not only for pure metal NPs (e.g., copper and gold) [93] but also metal fluoride materials [94]. Of particular interest is the use of this method to depose small $\mathrm{Cu}$ NPs onto $\mathrm{ZnO}$ nanomaterial already dispersed in the IL, creating a material with high potential in catalysis [18]. After evaporation, application of microwave irradiation was seen to fuse the two materials together. Indeed, the PVD method has high potential for the synthesis of a range of novel nanomaterials, as a range of materials may be directly evaporated into the ILs [95]. Binary materials may be achieved via simultaneous or consecutive evaporation of different materials and this presents a "clean" method as only substrate and IL are present, with no other regents needed nor by-products generated.

\section{Application of metal oxide NPs in ILs in catalysis}

\subsection{Hydrogenation}

One of the early examples by Dupont et al. described the application of small-sized $\mathrm{RuO}_{2} \mathrm{NPs}(2-3 \mathrm{~nm})$ prepared from $\mathrm{RuCl}_{3} \cdot 3 \mathrm{H}_{2} \mathrm{O}$ in 1- $n$-butyl-3-methylimidazolium hexafluorophosphate $\left(\mathrm{C}_{4} \mathrm{C}_{1} \mathrm{I} \cdot \mathrm{PF} \mathrm{F}_{6}\right)$ as a precursor for the catalytic hydrogenation of alkenes and arenes [32,33]. The NPs are suitable as recyclable catalysts for the hydrogenation of 1-hexene, 2,3-dimethyl-2-butene, cyclohexene, 1-methyl1-cyclohexene, benzene, toluene, and $p$-xylene under 4 bar $\mathrm{H}_{2}$ at $75^{\circ} \mathrm{C}$. The NPs could be recycled for 10 runs under solventless conditions for the hydrogenation of 1-hexene with a total turnover number (tTON) for exposed Ru atoms of 175,000 . Note that the catalysis under solventless conditions was at least three times faster than in the presence of ILs [32]. In further studies, it has been shown that high turnover frequencies (TOFs) $\left(>4000 \mathrm{~h}^{-1}\right)$ for solventless conditions (in the absence of IL stabilizers) can be obtained; however, the activity drops within four recycling steps below $700 \mathrm{~h}^{-1}$, yielding a tTON of 26,400. In contrast, the catalysis performed in a biphasic system in the presence of $\left[\mathrm{C}_{4} \mathrm{C}_{1} \mathrm{Im}\right]\left[\mathrm{PF}_{6}\right]$ gave TOFs between 1000 and $2000 \mathrm{~h}^{-1}$ over 17 runs, yielding tTON 110,000 [33]. This clearly shows the superior long-term stability of the IL-based catalyst system in comparison with the solventless system. Characterization of the NPs after catalysis with XRD and TEM and results from $\mathrm{CS}_{2}$ and $\mathrm{Hg}(0)$ poisoning experiments showed that the $\mathrm{RuO}_{2}$ were subsequently converted 
into $\mathrm{Ru}(0) \mathrm{NPs}$ during the hydrogenation reactions. Therefore, the $\mathrm{RuO}_{2}$ NPs act as precursor and the active species are $\mathrm{Ru}(0) \mathrm{NPs}$ in course of the hydrogenation reactions of alkenes and arenes. Similar observations were made by Liu et al. [50]. The authors prepared $\mathrm{Ru}(0) \mathrm{NPs}$ stabilized by IL on graphene. The analysis revealed the presence of ruthenium oxide, which was then subsequently reduced to $\mathrm{Ru}(0)$ during the hydrogenation of arenes, and in the case of benzene, they obtained TOFs more than $6000 \mathrm{~h}^{-1}$. In further studies by Dupont et al. using Ni NPs synthesized from $\left[\mathrm{Ni}(\mathrm{COD})_{2}\right]$ by hydrogenation in imidazolium ILs, it has been shown that the Ni NPs are capped with a NiO shell [35]. These $\mathrm{Ni} / \mathrm{NiO}$ particles are active for the hydrogenation of alkenes with a TOF of $91 \mathrm{~h}^{-1}$.

\subsection{Cross-coupling}

Corma et al. reported a protic imidazolium salt with a polyoxometalate anion (POM; $\left.\mathrm{H}_{5} \mathrm{PO}_{40} \mathrm{~V}_{2} \mathrm{Mo}_{10}\right)$ IL-POM ([C $\mathrm{C}_{1} \mathrm{I}_{4} \mathrm{HPO}_{40} \mathrm{~V}_{2} \mathrm{Mo}_{10}$ ) [41]. They reacted this IL-POM with $\mathrm{PdSO}_{4} \cdot 2 \mathrm{H}_{2} \mathrm{O}$ yielding $\left[\mathrm{C}_{4} \mathrm{C}_{1} \mathrm{Im}\right]_{4} \mathrm{Pd}_{0.5} \mathrm{PO}_{40} \mathrm{~V}_{2} \mathrm{Mo}_{10}$ (Pd-IL$\mathrm{POM})$, where Pd-O NPs $(\sim 2 \mathrm{~nm})$ were present on the Pd-ILPOM hybrid material. The Pd-O NPs are easily reduced to $\operatorname{Pd}(0)$, which could be evaluated for the Heck reaction in solvents of different polarity. Iodobenzene, styrene, and acrylate were used as model substrates and triethylamine as the base, at a temperature of $100^{\circ} \mathrm{C}$ with $100 \mathrm{ppm} \mathrm{Pd}$ loading. They obtained, for example, trans-stilbene with $100 \%$ selectivity at a conversion of $93 \%$ within $2 \mathrm{~h}$ and a corresponding TON of 15,000. The highest activity of course was obtained with polar solvents, such as DMF. They explained the higher activity in DMF in comparison with reactions in toluene with partial leaching of Pd into polar solvents such as DMF, as no leaching occurs in the former. Indeed, they observed in due course of the investigation that partial leaching occurs, but the leached Pd species are only responsible for $<10 \%$ of the conversion. Attempts to perform the reactions under solventless conditions to reduce the E-factor (amount of waste/amount of product) were less successful, as the activity was lower and higher reaction temperatures were needed to obtain good results. They also compared the Pd-IL-POM with the Pd-POM analogue and observed better results with the imidazolium-based system. The Pd-POM system was stable for only two runs, and already by the fourth run, the activity had dropped by $70 \%$; although no aggregation of Pd NPs occurred, the Pd-IL-POM showed a decreasing activity of $17 \%$ in the fourth run and $40 \%$ in the fifth run. They came to the conclusion that the catalyst material is more suitable for reactions that do not require a polar<smiles>[R][C]1C=C([N+](=O)[O-])C(C(=O)O)=CC1</smiles>

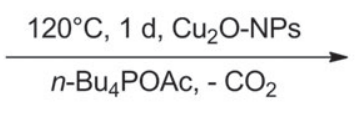<smiles>[R][C]1C=CC=CC1[N+](=O)[O-]</smiles>

$>10$ examples (moderate to high conversions)

Scheme $3 \mathrm{Cu}_{2} \mathrm{O}$ NPs catalyzed protodecarboxylation of 2-nitrobenzoic acid derivatives [13].

solvent (less leaching) or for gas-phase reactions to guarantee long-term stability.

\subsection{Decarboxylation}

Recently, it has been shown that $\mathrm{Cu}_{2} \mathrm{O}$ NPs in phosphonium acetate are recyclable (up to 10 runs) and active catalysts for the ligand-free and highly selective protodecarboxylation of 2-nitrobenzoic acids (Scheme 3) [13]. This reaction has been reported previously only with copper complexes and copper salts in the presence of ligands, but neither nanoscale catalysts nor recyclable catalysts have been studied. The highly polar IL makes the system feasible for multiphase catalysis, as the aromatic products such as nitrobenzene can easily be extracted with nonpolar solvents. The recycling can easily be achieved by simple reloading, hence the addition of benzoic acid substrates to the catalyst phase. Interestingly, the catalyst is selective for 2-nitrobenzoic acid derivatives; in a substrate scope of more than 20 substrates, only 2-nitrobenzoic acids were decarboxylated, highlighting the tolerance to various functional groups. In contrast, the absence of the nitro group ortho- to the acid group, no conversion was shown at all, even with highly electron-deficient groups. Therefore, the nitro-group is crucial for the NP-catalyzed decarboxylation, maybe acting as an anchoring ligand on the surface. Furthermore, this selectivity is maintained $(100 \%)$ even applying mixtures of electron-deficient benzoic acids.

\section{Application of bimetallic NPs in ILs in catalysis}

\subsection{Hydrogenation}

The first examples of bimetallic Pd-Au NPs dispersed in ILs were reported by Scott et al. Here, the authors 
performed a phase transfer of PVP-stabilized Pd-Au NPs into ILs and investigated the catalyst solutions in hydrogenation reactions examining different metal ratios [36]. The authors found that the activity as well as the reactivity in the catalytic hydrogenation is related to and can be controlled by the composition of the bimetallic material. The most effective ratio was found to be $\mathrm{Au} / \mathrm{Pd} 1: 3$, with TOFs up to $400 \mathrm{~h}^{-1}$, whereas monometallic Au NPs were inactive. These materials are recyclable in catalytic experiments with minimal loss in activity and the organic products and unreacted substrates were easily separated from the catalyst phase. The authors tested the bimetallic particles for the hydrogenation of allyl alcohol, 1,3-cyclooctadiene, trans-cinnamaldehyde, and 3-hexyn-1-ol (Figure 5).

They found that the higher efficiency is most likely related to electronic effects. In particular, electron density is withdrawn from the Pd regions to Au regions, resulting in an activation of Pd centers. These synergetic effects were observed in the case of the hydrogenation of the tested substrates. The lowest activity was found for trans-cinnamaldehyde and the best selectivity for the partial hydrogenation of 1,3-cyclooctadiene with $\mathrm{Au}-\mathrm{Pd}$ NPs (1:3). In further studies, Scott et al. showed that $\mathrm{Au}-\mathrm{Pd}$ NPs (ratio 1:3) synthesized directly in IL $\left[\mathrm{C}_{4} \mathrm{C}_{1} \mathrm{Im}\right]$ $\left[\mathrm{PF}_{6}\right]$ exhibited enhanced activity for the hydrogenation of allyl alcohol in the presence of small amounts of free 1-methylimidazole $(1.0 \mathrm{~mm})$ as a stabilizer [37]. Moreover, as already mentioned, the authors found that the long-term stability of the Au-Pd NPs in the presence of 1-methylimidazole was significantly higher than the stability of Au-Pd NPs in neat IL. This had a positive effect on the catalytic outcome, as under neat conditions a loss in catalytic activity was observed between $10 \mathrm{~min}$ and

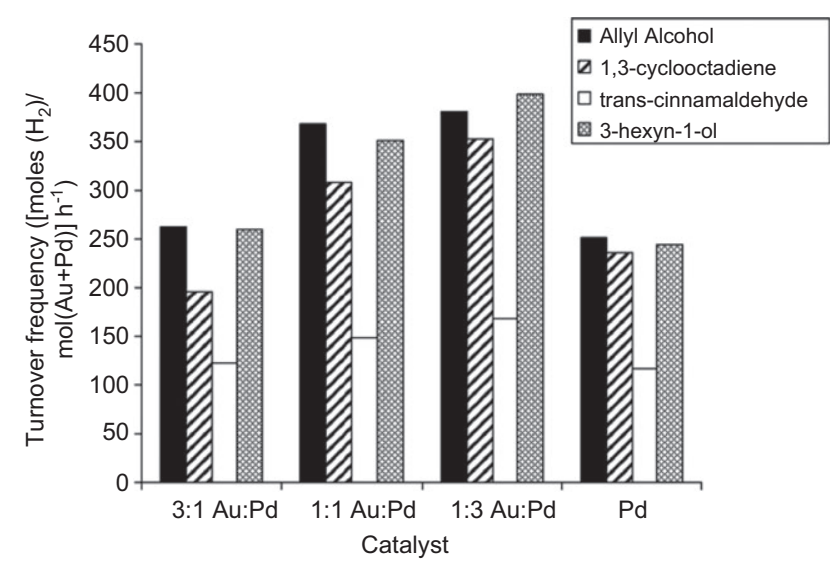

Figure 5 Comparison of the TOFs for the hydrogenation of allyl alcohol, 1,3-cyclooctadiene, trans-cinnamaldehyde, and 3-hexyn1-ol by Pd-Au NPs in $\left[\mathrm{C}_{4} \mathrm{C}_{1} \mathrm{Im}\right]\left[\mathrm{PF}_{6}\right]$. (C) Elsevier [36].
$1 \mathrm{~h}$, whereas 1-methylimidazole stabilized Au-Pd NPs showed pronounced activity after many recycles with consistent TOFs of $280 \mathrm{~h}^{-1}$. Similar results were obtained in $\left[\mathrm{C}_{4} \mathrm{C}_{1} \mathrm{Im}\right][\mathrm{OTf}]$; however higher 1-methylimidazole concentrations were necessary $(10-100 \mathrm{~mm})$. In continuation, the authors also compared other stabilizing agents for Au-Pd NPs in IL media [38]. Besides PVP- and 1-methylimidazole-stabilized Au-Pd NPs (1:3) in $\left[\mathrm{C}_{4} \mathrm{C}_{1} \mathrm{Im}\right]\left[\mathrm{PF}_{6}\right]$, they also used 1-(2-aminoethyl)-3-methylimidazolium hexafluorophosphate in the absence of secondary stabilizers. The smallest $(2.6 \pm 1.1 \mathrm{~nm})$, most stable, and catalytically active NP mixtures were those with PVP (1.4 mM) as added stabilizer in IL followed by (2) 1-methylimidazole (1.0 mM), (3) amine-functionalized IL (10 mM), and (4) neat $\left[\mathrm{C}_{4} \mathrm{C}_{1} \mathrm{Im}\right]\left[\mathrm{PF}_{6}\right]$. Interestingly, all systems showed coherent selectivity for the 1,3-cyclooctadiene hydrogenation to cyclooctene with $98 \%-99 \%$. The long-term stability of the Au-Pd NPs was tested with 3-buten-1-ol as a substrate. The best conditions used $0.22 \mathrm{~mm} \mathrm{Au}-\mathrm{Pd}$ (1:3), catalyst/substrate ratio 1:16,000 yielding a conversion of $100 \%$, and a TON specific to 1-butanol more than 10,000. The selectivity for 1-butanol was 68\%, accompanied by the isomerization products 2-buten-1-ol (22\%) and 1-butanal (10\%). The stability of the PVP-Au-Pd seems to be independent of the preparation methods (direct synthesis in IL and phase transfer into IL). Moreover, the bimetallic particles are superior to monometallic Pd NPs in terms of selectivity, the latter showing selectivities up to only $55 \%$. In conclusion, the PVP-Au-Pd NPs were the most stable and most catalytically active and presented the longest catalytic lifetime in comparison with those with other stabilizers.

Han et al. developed a hybrid catalyst material with bimetallic Au-Pd NPs [40]. The particles were obtained after a seeding growth process and were supported on crosslinked polymeric microspheres consisting of divinylbenzene and 1-vinyl-3-butyl imidazolium chloride ([C $\left.\left.\mathrm{C}_{4} \mathrm{VIm}\right][\mathrm{Cl}]\right)$. These Au-Pd NPs (PDVB-IL-Au/Pd) were evaluated for the hydrogenation of cyclohexene $(4 \mathrm{mmol})$ at $40^{\circ} \mathrm{C}$ along with Pd NPs and Au NPs. The monometallic Pd NP $\left(9.4 \times 10^{-5} \mathrm{mmol}\right)$ system was also active, whereas the systems with solely $\mathrm{Au}$ NPs were inactive. The most active system contained a Au/ Pd ratio of 1.2:1 $\left(9.4 \times 10^{-5} \mathrm{mmol}\right)$, which brought the reaction to completion four times faster ( $15 \mathrm{~min})$ than the monometallic Pd system (60 min). The authors related this to synergetic effects between $\mathrm{Au}$ and Pd. They interpreted this result also as an indication that bimetallic Au-Pd NPs were formed on the support. As the analysis of the material exhibited only very small lattice mismatches $(\mathrm{Pd}, \mathrm{Au}$, and $\mathrm{Au}-\mathrm{Pd}$ alloys), they believed that the enhanced activity was due to partial coverage of the Pd seeds with a gold shell. 


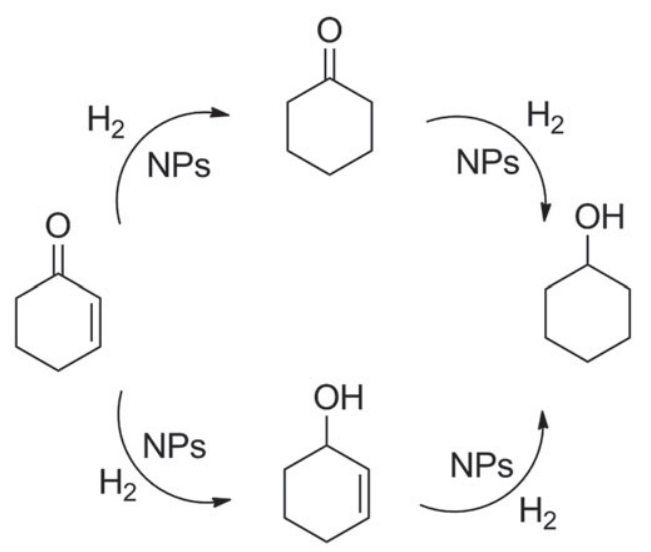

Scheme 4 Possible pathways and products during the hydrogenation of cyclohexenone. Adapted from Ref. [45].

Baiker et al. presented the application of bimetallic Fe-Ru NPs (Fe-Ru 9:1, Fe-Ru 3:1, and Fe-Ru 1:1) in imidazolium ILs [45], prepared via decarbonylation of the corresponding carbonyl precursors. The NPs were then evaluated for the selective hydrogenation of cyclohexenone to cyclohexanone (Scheme 4) at $50^{\circ} \mathrm{C}$ in batch reactor with an in operando ATR-IR probe and $\mathrm{CO}_{2}$ extraction unit.

The most active NP catalysts tested were the Fe-Ru NPs $(1.6 \mathrm{~nm})$ in a 1:1 ratio; remarkably, these were even more active than pure Ru NPs $(2.9 \mathrm{~nm})$. The activity could be further improved by expanding the IL media (1-butyl3-methylimidazolium tetrafluoroborate $\left.\left[\mathrm{C}_{4} \mathrm{C}_{1} \mathrm{Im}\right]\left[\mathrm{BF}_{4}\right]\right)$ with 60 bar $\mathrm{CO}_{2}$, which increased the rate fourfold. The selectivity under these conditions was more than $95 \%$ for cyclohexanone with a TOF of $300 \mathrm{~h}^{-1}$, and the catalyst could be recycled at least five times. Typically, the olefin is hydrogenated first, yielding cyclohexanone in the first step, whereas the formation of cyclohexenol was not detected in any experiment. Interestingly, at different $\mathrm{Ru} / \mathrm{Fe}$ ratios, the selectivity $(\sim 95 \%)$ for the ketone was kept constant for cyclohexanone, whereas the conversion rate increased with the Ru content. The selectivity was at least five times higher in comparison with pure Fe NPs. Likewise, the stability of the bimetallic particles in recycling experiments increased with $\mathrm{Ru}$ content. Pure Ru NPs gave also high conversions (94\%) but a lower selectivity (75\%) for cyclohexanone. One particular difference between the pure $\mathrm{Ru}$ catalyst and the bimetallic Fe-containing catalysts is that the latter suffered from deactivation in recycling experiments after $\mathrm{CO}_{2}$ extraction. In contrast, the monometallic $\mathrm{Ru}$ NPs showed increasing conversion and selectivity upon reuse. Neither effects (i.e., the enhanced activity of $\mathrm{Ru}$ NPs and deactivation of bimetallic Ru-Fe NPs) are fully understood. The best activity for the Ru-Fe NPs $(1.6 \mathrm{~nm})$ with a 1:1 ratio is most likely related to the smaller size in comparison with the Ru NPs $(2.9 \mathrm{~nm})$. In conclusion, the authors found beneficial effects on the $\mathrm{CO}_{2}$ addition: (I) the reaction rate increased four times due to improved mass transfer and hydrogen solubility, (II) the selectivity toward cyclohexanone in the presence of $\mathrm{CO}_{2}$ increased also four times than in the absence of $\mathrm{CO}_{2}$ and no cyclohexanol was formed, (III) the product separation is facilitated by extraction with $\mathrm{sc} \mathrm{CO}_{2}$, and (IV) $\mathrm{scCO}_{2}$ improves and tunes the properties of the IL.

\subsection{Hydrogenolysis}

Han and his research group reported a series of bimetallic $\mathrm{Ru}-\mathrm{Cu}$ catalysts modified with a guanidinium salt (1,1,3,3-tetramethylguanidinium lactate; TMGL) on BEN toward the chemical transformation of glycerol [39]. They performed hydrogenolysis of glycerol in aqueous solution between $190^{\circ} \mathrm{C}$ and $240^{\circ} \mathrm{C}$ and pressures of 2.5 to $10 \mathrm{MPa}$ with different catalyst loadings obtaining variation in selectivity, activity, and yield. The best results were obtained with $\mathrm{Ru}-\mathrm{Cu} / \mathrm{TMG}-\mathrm{BEN}(\mathrm{Ru} 3 \mathrm{wt} . \%, \mathrm{Ru} / \mathrm{Cu}$ 3:1). Here, $100 \%$ glycerol conversion and $85 \%$ yield of 1,2-propanediol (1,2-PDO) could be achieved at $230^{\circ} \mathrm{C}$ and pressure of $8 \mathrm{MPa}$. Moreover, the catalyst was recyclable for at least five times with similar activity and selectivity. As by-products, the following compounds could be detected: 1,3-propanediol (1,3-PDO), ethylene glycol (EG), 1-propanol (1-PO), 2-propanol (2-PO), ethanol, methanol, methane, and $\mathrm{CO}_{2}$. The authors concluded that the IL is crucial for the stability and recyclability of the catalyst. The selectivity toward 1,2-PDO is most likely related to the presence of copper as catalyst poison, as pure Ru catalysts and other $\mathrm{Ru} / \mathrm{Cu}$ ratios were less efficient in terms of selectivity rather than activity.

\subsection{Oxidation}

In their ongoing studies about bimetallic Au-Pd NPs in ILs, Scott et al. found that these materials are suitable for the oxidation of $\alpha, \beta$-unsaturated alcohols (Scheme 5) [46]. Besides PVP-stabilized bimetallic particles, the authors evaluated tetraalkylphosphonium chloride ILs as stabilizers during the sequential reduction of the metal precursors yielding a variety of core-shell particles. The solely $\mathrm{Au}$ NPs were found to be inefficient, but Au NPs mixed with Pd(II) salts served also as catalyst for the oxidation of unsaturated alcohols under base-free conditions with oxygen as single oxidant at $60^{\circ} \mathrm{C}$. 


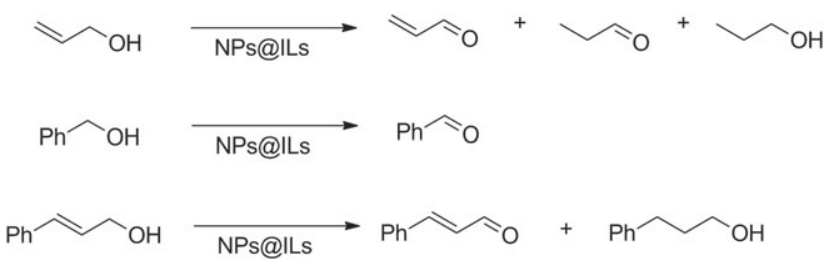

Scheme 5 Possible products during the alcohol oxidation, possibly accompanied by isomerization and transfer-hydrogenation (selected examples of the substrate scope).

Adapted from Ref. [46].

Analysis of the catalyst material after usage showed that the $\mathrm{Pd}(\mathrm{II})$ salt was reduced in situ during the alcohol oxidation; also, particle size and the morphology changed after catalysis most likely due to Ostwald ripening and the redox process. The IL acts probably in a twofold manner; the chloride interacts with the particle surface, whereas the hydrocarbon side-chains of the cation provide steric protection. The authors tested the IL-based system for its recyclability to gauge whether the IL system (vacuum extraction) is advantageous in comparison with the water-based system (liquid-liquid extraction). The authors explain the higher activity in IL in comparison with the water system as halide ions provoking oxidative etching of $\mathrm{Pd}$ and $\mathrm{Au}$ in the presence of oxygen. The partially oxidized particles undergo reduction in a stoichiometric reaction with the alcohols, oxidizing the alcohols in a redox process. This has been observed to a greater extent in the presence of the halide ILs rather than in the aqueous system with a low halide concentration. The authors tested allyl alcohol, benzyl alcohol, cinnamyl alcohol. and crotyl alcohol with substrate/catalyst ratios of 5000:1, with the phosphonium IL as stabilizer for Pd NPs, Au-Pd NPs, and Au NPs/Pd(II). In contrast, in the aqueous system, a substrate/catalyst ratio of 500:1 was applied. The best conversions were obtained with Pd NPs and Au NPs/Pd(II) catalysts. The Au-Pd NPs (1:3) exhibited a variation in terms of conversion and selectivity for the aldehyde. In general, the conversions in the IL systems are much higher than in the aqueous system. Moreover and unsurprisingly, the aromatic substrates such as benzyl alcohol and cinnamyl alcohol show very high conversions and high selectivity. For allyl alcohol, the major product is 1-propanal. For crotyl alcohol, the conversion is similar to those in the aqueous system but with lower selectivity. To exclude the influence of the substrate/catalyst ratio, the experiment was repeated with a ratio of 500:1 as performed in water, finally giving similar results, no matter the reaction medium (IL vs. water). In summary, one can conclude that the Au-Pd NPs are not superior to the Pd NPs regarding the activity and selectivity for the alcohol oxidation, which is probably related to the oxidative etching of both particle types in halide ILs. Therefore, Au shows no effect as promoter for the Pd-catalyzed oxidation of alcohol in the IL system, which remains contradictory to the previously discussed hydrogenation reactions with Au-Pd NPs in IL media [36-38].

\subsection{Dehalogenation}

Yan et al. generated spherical and small-sized (3-5 nm) monometallic and bimetallic Au-Pd NPs in different metal ratios $\left(\mathrm{Au}, \mathrm{Au}_{0.8} \mathrm{Pd}_{0.2}, \mathrm{Au}_{0.5} \mathrm{Pd}_{0.5}, \mathrm{Au}_{0.2} \mathrm{Pd}_{0.8}\right.$, and $\mathrm{Pd}$ $\mathrm{NPs}$ ) in the functionalized IL $\left[\mathrm{C}_{2} \mathrm{OHIm}\right]\left[\mathrm{NTf}_{2}\right]$ using the acetate anion $[13,90]$ as reducing agent of the metal precursors $\mathrm{Pd}(\mathrm{OAc})_{2}$ and $\mathrm{Au}(\mathrm{OAc})_{3}$ [55]. Similar to reports by Scott et al. [36-38, 46, 96], they observed superior catalytic activity for bimetallic Au-Pd NPs in comparison with weakly active monometallic Pd NPs and inactive Au NPs. The major difference between Yan's and Scott's results are that the former found a novel route to bimetallic NPs, which have Pd enrichment on the surface. As a model system to determine the catalytic activity of the series of bimetallic NPs, they investigated the dehalogenation of arenes under hydrogen atmosphere. The authors stated that the enhanced activity of Au-Pd NPs with a Pd-enriched surface and Au core are also related to a charge transfer from $\mathrm{Pd}$ to $\mathrm{Au}$ resulting in a more efficient elimination. The hydrogenation of 2-chloronitrobenzene with Pd NPs gave as major product (85\%) 2-chloroaniline, whereas, with $\mathrm{Au}_{0.8} \mathrm{Pd}_{0.2} \mathrm{NPs}$, the major product is cyclohexylamine (Scheme 6).

Also interesting is the observation of arene hydrogenation with a Pd-based nanoscale catalyst; usually, Pd is inactive for arene hydrogenation in ILs, and other metals, such as Rh, Pt, Ir, and Ru, are necessary to achieve this type of hydrogenation. Dehalogenation was also observed for bromoarenes such as bromotoluene derivatives. Again,

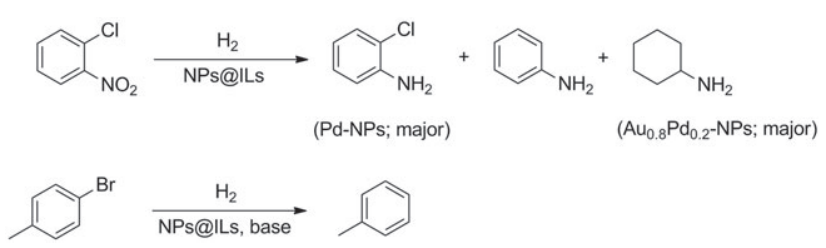

Scheme 6 Au-Pd NPs catalyzed dehalogenation under base-free conditions (top) and in the presence of base (bottom).

Adapted from Ref. [55]. 


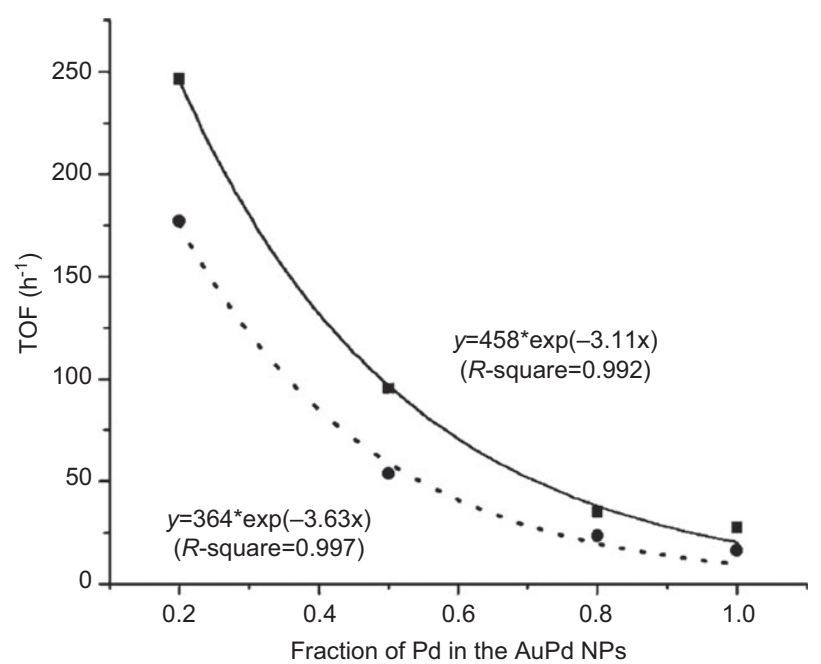

Figure 6 TOF as function of the Pd fraction in bimetallic NPs. (c) Wiley-VCH [55].

the bimetallic NPs were more active than Pd NPs, whereas pure $\mathrm{Au}$ NPs were inactive. The hydrogenation of the aromatic ring was suppressed by the addition of potassium carbonate as a base; thus, for 4-bromotoluene, the selectivity of toluene was $100 \%$ for all NPs and the activity followed the trend: $\mathrm{Au}_{0.8} \mathrm{Pd}_{0.2}\left(71 \%\right.$; TOF $\left.=177 \mathrm{~h}^{-1}\right)>\mathrm{Au}_{0.5} \mathrm{Pd}_{0.5}$ (54\%; TOF $\left.=54 \mathrm{~h}^{-1}\right)>\mathrm{Au}_{0.2} \mathrm{Pd}_{0.8}\left(38 \%\right.$; TOF $\left.=24 \mathrm{~h}^{-1}\right)>\mathrm{Pd} \mathrm{NPs}$ $\left(32 \%\right.$; TOF $\left.=16 \mathrm{~h}^{-1}\right)$. For 3,5-dibromotoluene, the best conversion (98.5\%), TOF (246 $\left.\mathrm{h}^{-1}\right)$, and selectivity for toluene (93\%) were obtained with $\mathrm{Au}_{0.8} \mathrm{Pd}_{0.2}$ NPs. Again, the conversion and selectivity is related to the molar fraction of Pd, where monometallic Pd NPs showed lowest conversion (55\%) and a selectivity of $38 \%$ following the same trend with $\mathrm{Au}_{0.8} \mathrm{Pd}_{0.2}$ as the most active. The plot (Figure 6) of increasing catalytic activity with increasing $\mathrm{Au}$ fraction (hence decreasing Pd fraction) shows a clear trend. This trend might be attributed to enrichment of $\mathrm{Pd}$ at the NP surface and the electronic changes to the Pd atoms induced by electronegative $\mathrm{Au}$, resulting in more electropositive Pd on the NP surface. In conclusion, the best results can be obtained with a low $\mathrm{Pd}$ fraction $\left(\mathrm{Au}_{0.8} \mathrm{Pd}_{0.2}\right)$, giving more electropositive $\mathrm{Pd}$, which has a higher catalytic activity for dehalogenation.

\section{Conclusion and summary}

Although the subject remains in its infancy, the results gathered herein show that ILs are suitable media for the synthesis of well-defined metal oxide and bimetallic NPs, which may be used for catalytic applications in multiphase systems alongside others. Similar to monometallic metal(0) NPs, the ILs serve as reaction media and stabilization agent for the small-sized NPs, and no additional capping agents are required. Thus, the particle surface remains accessible for catalytic reactions. Also, the use of ILs, thanks to their unique and tailorable properties, opens the door to new and improved synthesis procedures for these NPs. In addition, varying the IL nature and reaction conditions can lead to different sizes morphologies and, in the case of bimetallic NPs, can dictate the formation of alloy versus core-shell NPs.

Metal oxide and bimetallic NPs dispersed in ILs are interesting classes of catalytically active materials and recyclable catalysts for reactions in multiphase systems. The selectivity of these materials shows that they are suitable as multisite catalysts and are superior in certain cases to monometallic catalyst systems. Among the promising perspectives of these materials are their tuneable and different physicochemical properties in comparison with monometallic metal(0) NPs in ILs. The long-term stability can be estimated as similar to those of metal(0) NPs in ILs, but until now, there are only few examples for comparative studies. Again, functional groups on IL sidechains play a key role for the stabilization and activity of the nanoscale catalysts.

So far, the best understood and studied system in ILs is the bimetallic Au-Pd NPs system. In general, Au-Pd NPs are more active than the corresponding monometallic NPs catalysts for hydrogenation, oxidation, and dehalogenation reactions. This is probably related to electronic effects; $\mathrm{Au}$ withdraws electron density from the Pd, thus making the catalytically active Pd centers more electropositive and more catalytically active. Bimetallic NPs with Ru as the catalytically active center together with $\mathrm{Cu}$ or $\mathrm{Fe}$ show higher selectivity in hydrogenation and hydrogenolysis reactions. In contrast to Au-Pd NPs, where Au enhances the catalytic activity of Pd, in the case of Ru-Cu and Ru-Fe, the copper and iron play more a role as catalyst poison to decrease the catalytic activity of Ru but can enhance the selectivity for partial hydrogenation for instance.

The rare examples of metal oxide NPs in ILs show that, in most cases, the metal oxides (or oxide shells) are converted into metal(0) NPs during the catalytic reactions, such as in hydrogenation $(\mathrm{Ru}, \mathrm{Ni})$ or cross-coupling (Pd) reactions. On the contrary, the protodecarboxylation catalyzed by $\mathrm{Cu}_{2} \mathrm{O}$ NPs in ILs is known to be catalyzed by $\mathrm{Cu}(\mathrm{I})$ species rather than $\mathrm{Cu}(0)$ or $\mathrm{Cu}(\mathrm{II})$ species. For metal oxides and even more so for bimetallic NPs in ILs, there are promising applications throughout the vast field of multiphase catalysis due to (1) enhanced activity (electronic effects), (2) enhanced selectivity (poisoning), 
and (3) enhanced recyclability (decreased leaching and enhanced catalyst separation).

Acknowledgements: PCS acknowledges Dr. C. Santini and Prof. A.-V. Mudring for allowance to participate in this review, and PSC thanks Prof. Mudring for past, current and future support in working towards a successful independent career.

Received April 11, 2013; accepted June 5, 2013; previously published online July 23, 2013

\section{References}

[1] Schmid G. Nanoparticles from Theory to Application, Wiley-VCH: Weinheim, 2004.

[2] Bönnemann H, Nagabhushana KS. In Surface and Nanomolecular Catalysis, Richards RM, Ed., CRC Press: Boca Raton, 2006, pp. 63-94.

[3] Astruc D, Lu F, Aranzaes JR. Nanoparticles as recyclable catalysts: the Frontier between homogeneous and heterogeneous catalysis. Angew. Chem. Int. Ed. 2005, 44, 7852-7872.

[4] Astruc D. In Nanoparticles and Catalysis, Astruc D, Ed., Wiley-VCH: Weinheim, 2007.

[5] Roucoux A, Schulz J, Patin H. Reduced transition metal colloids:? a novel family of reusable catalysts?. Chem. Rev. 2002, 102, 3757-3778.

[6] Roucoux A, Nowicki A, Philippot K. In Nanoparticles and Catalysis, Astruc D, Ed., Wiley-VCH: Weinheim, 2007, pp. 349-390.

[7] Roucoux A, Philippot K. In Handbook of Homogeneous Hydrogenation, Vries JGd, Elsevier CJ, Eds., Wiley-VCH: Weinheim, 2007, pp. 217-256.

[8] Valden M, Lai X, Goodman DW. Onset of catalytic activity of gold clusters on titania with the appearance of nonmetallic properties. Science 1998, 281, 1647-1650.

[9] Bell AT. The impact of nanoscience on heterogeneous catalysis. Science 2003, 299, 1688-1691.

[10] Bönnemann H, Nagabhushana KS. In Metal Nanoclusters in Catalysis and Materials Science: The Issue of Size-Control, Corain B, Schmid G, Toshima N, Eds., Elsevier: Amsterdam, 2007, pp. 21-48.

[11] Chaudret B. Synthesis and surface reactivity of organometallic nanoparticles. Top. Organomet. Chem. 2005, 16, 233-259.

[12] Amiens C, Chaudret B. Organometallic synthesis of nanoparticles. Mod. Phys. Lett. B 2007, 21, 1133-1141.

[13] Kessler MT, Gedig C, Sahler S, Wand P, Robke S, Prechtl MHG. Recyclable nanoscale copper(I) catalysts in ionic liquid media for selective decarboxylative $\mathrm{C}-\mathrm{C}$ bond cleavage. Catal. Sci. Technol. 2013, 3, 992-1001.

[14] Dupont J, Scholten JD. On the structural and surface properties of transition-metal nanoparticles in ionic liquids. Chem. Soc. Rev. 2010, 39, 1780-1804.

[15] Scholten JD, Leal CB, Dupont J. Transition metal nanoparticle catalysis in ionic liquids. Acs Catal. 2012, 2, 184-200.

[16] Wender H, Migowski P, Feil AF, de Oliveira LF, Prechtl MHG, Leal R, Machado G, Teixeira SR, Dupont J. On the formation of anisotropic gold nanoparticles by sputtering onto a nitrile functionalised ionic liquid. Phys. Chem. Chem. Phys. 2011, 13, 13552-13557.

[17] Wender H, de Oliveira LF, Migowski P, Feil AF, Lissner E, Prechtl MHG, Teixeira SR, Dupont J. Ionic liquid surface composition controls the size of gold nanoparticles prepared by sputtering deposition. J. Phys. Chem. C 2010, 114, 11764-11768.

[18] Richter K, Birkner A, Mudring AV. Stabilizer-free metal nanoparticles and metal-metal oxide nanocomposites with long-term stability prepared by physical vapor deposition into ionic liquids. Angew. Chem. Int. Ed. 2010, 49, 2431-2435.

[19] Helgadottir IS, Arquillière PP, Bréa P, Santini CC, Haumesser PH, Richter K, Mudring AV, Aouine M. Synthesis of bimetallic nanoparticles in ionic liquids: Chemical routes vs physical vapor deposition. Microelectronic Eng. 2013, 107, 229-232.

[20] Krossing I, Slattery JM, Daguenet C, Dyson PJ, Oleinikova A, Weingartner $\mathrm{H}$. Why are ionic liquids liguid? a simple explanation based on lattice and solvation energies. J. Am. Chem. Soc. 2006, 128, 13427-13434.

[21] Wasserscheid P, Welton T. Ionic Liquids in Synthesis, Wiley-VCH: Weinheim, 2007.

[22] Welton T. Room-temperature ionic liquids. solvents for synthesis and catalysis. Chem. Rev. 1999, 99, 2071-2084.

[23] Welton T. Ionic liquids in catalysis. Coord. Chem. Rev. 2004, 248, 2459-2477.

[24] Hallett JP, Welton T. Room-temperature ionic liquids: solvents for synthesis and catalysis. 2. Chem. Rev. 2011, 111, 3508-3576.

[25] Prechtl MHG, Scholten JD, Dupont J. Tuning the selectivity of ruthenium nanoscale catalysts with functionalised ionic liquids: hydrogenation of nitriles. J. Mol. Catal. A Chem. 2009, 313, 74-78.

[26] Darwich W, Gedig C, Prechtl MHG, Santini CC. Dihydroxyl Imidazolium Ionic Liquids: Solvent, Ligand and Reducing Agent in Metallic Nanoparticles Synthesis. Proceedings of the 5th Congress of Ionic Liquids, 2013, p. 44.

[27] Prechtl MHG, Campbell PS, Scholten JD, Fraser GB, Machado G, Santini CC, Dupont J, Chauvin Y. Imidazolium ionic liquids as promoters and stabilising agents for the preparation of metal $(0)$ nanoparticles by reduction and decomposition of organometallic complexes. Nanoscale 2010, 2, 2601-2606.

[28] Canongia Lopes JN, Padua AAH. Nanostructural organization in ionic liquids. J. Phys. Chem. B 2006, 110, 3330-3335.

[29] Wang Y, Izvekov S, Yan T, Voth GA. Multiscale coarse-graining of ionic liquids. J. Phys. Chem. B 2006, 110, 3564-3575.

[30] Gutel T, Santini CC, Philippot K, Padua A, Pelzer K, Chaudret B, Chauvin Y, Basset JM. Organized 3D-alkyl imidazolium ionic liquids could be used to control the size of in situ generated ruthenium nanoparticles?. J. Mater. Chem. 2009, 19, 3624-3631. 
[31] Prechtl MHG, Scholten JD, Dupont J. Carbon-carbon cross coupling reactions in ionic liquids catalysed by palladium metal nanoparticles. Molecules 2010, 15, 3441-3461.

[32] Rossi LM, Dupont J, Machado G, Fichtner PFP, Radtke C, Baumvol IJR, Teixeira SR. Ruthenium dioxide nanoparticles in ionic liquids: synthesis, characterization and catalytic properties in hydrogenation of olefins and arenes. J. Braz. Chem. Soc. 2004, 15, 904-910.

[33] Rossi LM, Machado G, Fichtner PFP, Teixeira SR, Dupont J. On the use of ruthenium dioxide in 1-n-butyl-3-methylimidazolium ionic liquids as catalyst precursor for hydrogenation reactions. Catal. Lett. 2004, 92, 149-155.

[34] Miao SD, Liu ZM, Zhang ZF, Han BX, Miao Z), Ding KL, An GM. Ionic liquid-assisted immobilization of rh on attapulgite and its application in cyclohexene hydrogenation. J. Phys. Chem. C 2007, 111, 2185-2190.

[35] Migowski P, Machado G, Texeira SR, Alves MCM, Morais ], Traverse A, Dupont J. Synthesis and characterization of nickel nanoparticles dispersed in imidazolium ionic liquids. Phys. Chem. Chem. Phys. 2007, 9, 4814-4821.

[36] Dash P, Dehm NA, Scott RW]. Bimetallic PdAu nanoparticles as hydrogenation catalysts in imidazolium ionic liquids. J. Mol. Catal. A Chem. 2008, 286, 114-119.

[37] Dash P, Scott RWJ. 1-Methylimidazole stabilization of gold nanoparticles in imidazolium ionic liquids. Chem. Commun. 2009, 812-814.

[38] Dash P, Miller SM, Scott RWJ. Stabilizing nanoparticle catalysts in imidazolium-based ionic liquids: a comparative study. J. Mol. Catal. A Chem. 2010, 329, 86-95.

[39] Jiang T, Zhou YX, Liang SG, Liu HZ, Han BX. Hydrogenolysis of glycerol catalyzed by Ru-Cu bimetallic catalysts supported on clay with the aid of ionic liquids. Green Chem. 2009, 11, 1000-1006.

[40] Hu BJ, Wu TB, Ding KL, Zhou XS, Jiang T, Han BX. Seeding growth of $\mathrm{Pd} / \mathrm{Au}$ bimetallic nanoparticles on highly cross-linked polymer microspheres with ionic liquid and solvent-free hydrogenation. J. Phys. Chem. C $2010,114,3396-3400$.

[41] Corma A, Iborra S, Xamena F, Monton R, Calvino JJ, Prestipino C. Nanoparticles of Pd on hybrid polyoxometalateionic liquid material: synthesis, characterization, and catalytic activity for heck reaction. J. Phys. Chem. C 2010, 114, 8828-8836.

[42] Snyder J, Fujita T, Chen MW, Erlebacher J. Oxygen reduction in nanoporous metal-ionic liquid composite electrocatalysts. Nat. Mater. 2010, 9, 904-907.

[43] Lee JS, Lee T, Song HK, Cho J, Kim BS. Ionic liquid modified graphene nanosheets anchoring manganese oxide nanoparticles as efficient electrocatalysts for $\mathrm{Zn}$-air batteries. Energy Environ. Sci. 2011, 4, 4148-4154.

[44] Valizadeh $\mathrm{H}$, Azimi AA. $\mathrm{ZnO} / \mathrm{MgO}$ containing $\mathrm{ZnO}$ nanoparticles as a highly effective heterogeneous base catalyst for the synthesis of $4 \mathrm{H}$-pyrans and coumarins in $\left[\mathrm{bmim} \mathrm{BF}_{4 .}\right.$ J. Iran. Chem. Soc. 2011, 8, 123-130.

[45] Andanson JM, Marx S, Baiker A. Selective hydrogenation of cyclohexenone on iron-ruthenium nano-particles suspended in ionic liquids and $\mathrm{CO}_{2}$-expanded ionic liquids. Catal. Sci. Technol. 2012, 2, 1403-1409.

[46] Maclennan A, Banerjee A, Scott RWJ. Aerobic oxidation of a, $\beta$-unsaturated alcohols using sequentially-grown AuPd nanoparticles in water and tetraalkylphosphonium ionic liquids. Catal. Today 2012, 207, 170-179.
[47] Safavi A, Momeni S, Tohidi M. Silver-palladium nanoalloys modified_carbon ionic liquid electrode with enhanced electrocatalytic activity towards formaldehyde oxidation. Electroanalysis 2012, 24, 1981-1988.

[48] Souza BS, Pinho DMM, Leopoldino EC, Suarez PAZ, Nome F. Selective partial biodiesel hydrogenation using highly active supported palladium nanoparticles in imidazolium-based ionic liquid. Appl. Catal. A Gen. 2012, 433, 109-114.

[49] Suzuki S, Suzuki T, Tomita Y, Hirano M, Okazaki K, Kuwabata S, Torimoto T. Compositional control of AuPt nanoparticles synthesized in ionic liquids by the sputter deposition technique. Crystengcomm 2012, 14, 4922-4926.

[50] Xiao WJ, Sun ZY, Chen S, Zhang HY, Zhao YF, Huang CL, Liu ZM. Ionic liquid-stabilized graphene and its use in immobilizing a metal nanocatalyst. Rsc Adv. 2012, 2, 8189-8193.

[51] Zhao HD, Yu CZ, You HJ, Yan SC, Guo Y, Ding BJ, Song XP. A green chemical approach for preparation of $\mathrm{Pt}_{-x} \mathrm{Cu}_{y}$ nanoparticles with a concave surface in molten salt for methanol and formic acid oxidation reactions. J. Mater. Chem. 2012, 22, 4780-4789.

[52] Zhou FL, Izgorodin A, Hocking RK, Spiccia L, MacFarlane DR. Electrodeposited $\mathrm{mnO}$ films from ionic liquid for electrocatalytic water oxidation. Adv. Energy Mater. 2012, 2, 1013-1021.

[53] Safavi A, Kazemi H, Momeni S, Tohidi M, Khanipour MP. Facile electrocatalytic oxidation of ethanol using $\mathrm{Ag} / \mathrm{Pd}$ nanoalloys modified carbon ionic liquid electrode. Int. J. Hydrogen Energy 2013, 38, 3380-3386.

[54] Sharghi H, Ebrahimpourmoghaddam S, Memarzadeh R, Javadpour S. Tin oxide nanoparticles (NP-SnO $)$ : preparation, characterization and their catalytic application in the Knoevenagel condensation. J. Iran. Chem. Soc. 2013, 10, 141-149.

[55] Yuan X, Sun G, Asakura H, Tanaka T, Chen X, Yuan Y, Laurenczy G, Kou Y, Dyson PJ, Yan N. Development of palladium surface-enriched heteronuclear Au-Pd nanoparticle dehalogenation catalysts in an ionic liquid. Chem. Eur. J. 2013, 2013 , 1227-1234.

[56] Alias A, Hamzah N, Yarmo MA. Hydrogenolysis of glycerol to propanediols over nano-ru/c catalyst with ionic liquid addition. Adv. Mater. Res. Switz. 2011, 173, 49-54.

[57] Chen W, Chen SW. Iridium-platinum alloy nanoparticles: composition-dependent electrocatalytic activity for formic acid oxidation. J. Mater. Chem. 2011, 21, 9169-9178.

[58] Xu JL, Zhang C, Wang XG, Ji H, Zhao CC, Wang Y, Zhang ZH. Fabrication of bi-modal nanoporous bimetallic Pt-Au alloy with excellent electrocatalytic performance towards formic acid oxidation. Green Chem. 2011, 13, 1914-1922.

[59] Kumar CCSR, Vol. 2: Nanostructured Oxides, Wiley-VCH: Weinheim, 2009.

[60] Bilecka I, Niederberger M. New developments in the nonaqueous and/or non-hydrolytic sol-gel synthesis of inorganic nanoparticles. Electrochim. Acta 2010, 55, 7717-7725.

[61] Djerdj I, Arcon D, Jaglicic Z, Niederberger M. Nonaqueous synthesis of metal oxide nanoparticles: Short review and doped titanium dioxide as case study for the preparation of transition metal-doped oxide nanoparticles. J. Solid State Chem. 2008, 181, 1571-1581.

[62] Niederberger M, Garnweitner G. Organic reaction pathways in the nonaqueous synthesis of metal oxide nanoparticles. Chem. Eur. J. 2006, 12, 7282-7302. 
[63] Alammar T, Birkner A, Mudring A-V. Ultrasound-assisted synthesis of cuo nanorods in a neat room-temperature ionic liquid. Eur. J. Inorg. Chem. 2009, 2765-2768.

[64] Alammar T, Birkner A, Shekhah 0, Mudring A-V. Sonochemical preparation of $\mathrm{TiO} 2$ nanoparticles in the ionic liquid 1-(3-hydroxypropyl)-3-methylimidazoliumbis(trifluoromethylsulfonyl)amide. Mater. Chem. Phys. 2010, 120, 109-113.

[65] Alammar T, Mudring A-V. Facile preparation of $\mathrm{Ag} / \mathrm{ZnO}$ nanoparticles via photoreduction. J. Mater. Sci. 2009, 44, 3218-3222.

[66] Hou X, Zhou F, Sun Y, Liu W. Ultrasound-assisted synthesis of dentritic ZnO nanostructure in ionic liquid. Mater. Lett. 2007, 61, 1789-1792.

[67] Alammar T, Mudring A-V. Sonochemical synthesis of OD, 1D, and 2D zinc oxide nanostructures in ionic liquids and their photocatalytic activity. ChemSusChem 2011, 4, 1796-1804.

[68] Alammar T, Shekhah O, Wohlgemuth J, Mudring AV. Ultrasoundassisted synthesis of mesoporous $\beta-\mathrm{Ni}(\mathrm{OH}) 2$ and $\mathrm{NiO}$ nano-sheets using ionic liquids. J. Mater. Chem. 2012, 22, 18252-18260.

[69] Wang W-W, Zhu Y-J. Shape-controlled synthesis of zinc oxide by microwave heating using an imidazolium salt. Inorg. Chem. Commun. 2004, 7, 1003-1005.

[70] Li KF, Luo H, Ying TK. One-step, solid-state reaction to ZnO nanoparticles in the presence of ionic liquid. Mater. Sci. Semicond. Proc. 2011, 14, 184-187.

[71] Zhu H, Huang J-F, Pan Z, Dai S. Ionothermal synthesis of hierarchical zno nanostructures from ionic-liquid precursors. Chem. Mater. 2006, 18, 4473-4477.

[72] Avellaneda RS, Ivanova S, Sanz O, Romero-Sarria F, Centeno MA, Odriozola JA. Ionic liquid templated $\mathrm{TiO}_{2}$ nanoparticles as a support in gold environmental catalysis. Appl. Catal. B Environ. 2009, 93, 140-148.

[73] Jacob DS, Bitton L, Grinblat J, Felner I, Koltypin Y, Gedanken A. Are ionic liquids really a boon for the synthesis of inorganic materials? A general method for the fabrication of nanosized metal fluorides. Chem. Mater. 2006, 18, 3162-3168.

[74] Lee CM, Jeong HJ, Lim ST, Sohn MH, Kim DW. Synthesis of iron oxide nanoparticles with control over shape using imidazoliumbased ionic liquids. Acs Appl. Mater. Interf. 2010, 2, 756-759.

[75] Zhao JB, Wu LL, Zou K. Fabrication of hollow mesoporous NiO hexagonal microspheres via hydrothermal process in ionic liquid. Mater. Res. Bull. 2011, 46, 2427-2432.

[76] Kandjani AE, Tabriz MF, Pourabbas B. Sonochemical synthesis of $\mathrm{ZnO}$ nanoparticles: The effect of temperature and sonication power. Mater. Res. Bull. 2008, 43, 645-654.

[77] Wei YL, Chang PC. Characteristics of nano zinc oxide synthesized under ultrasonic condition. J. Phys. Chem. Solids 2008, 69, 688-692.

[78] Campbell PS, Lorbeer C, Cybinska J, Mudring A-V. Adv. Funct. Mater. 2013, 23, 2924-2931.

[79] Lorbeer C, Cybinska J, Mudring A-V. Facile preparation of quantum cutting $\mathrm{GdF}_{3}$ : $\mathrm{Eu}^{3+}$ nanoparticles from ionic liquids. Chem. Commun. 2010, 46, 571-573.

[80] Campbell PS, Prechtl MHG, Santini CC, Haumesser P-H. Ruthenium nanoparticles in ionic liquids - a saga. Curr. Org. Chem. 2013, 17, 414-429.

[81] Murray JL, Massalski TB, Bennett LH, Baker H. Binary Alloy Phase Diagrams, Vol. I and II, ASM International: Cleveland, $\mathrm{OH}, 1986$.
[82] Kramer J, Redel E, Thomann R, Janiak C. Use of ionic liquids for the synthesis of iron, ruthenium, and osmium nanoparticles from their metal carbonyl precursors. Organometallics 2008, 27, 1976-1978.

[83] Redel E, Thomann R, Janiak C. Use of ionic liquids (ILs) for the IL-anion size-dependent formation of $\mathrm{Cr}$, Mo and $\mathrm{W}$ nanoparticles from metal carbonyl $\mathrm{M}(\mathrm{CO})_{6}$ precursors. Chem. Commun. 2008, 1789-1791.

[84] Vollmer C, Janiak C. Naked metal nanoparticles from metal carbonyls in ionic liquids: easy synthesis and stabilization. Coord. Chem. Rev. 2011, 255, 2039-2057.

[85] Vollmer C, Redel E, Abu-Shandi K, Thomann R, Manyar H, Hardacre C, Janiak C. Microwave irradiation for the facile synthesis of transition-metal nanoparticles (NPs) in ionic liquids (ILs) from metal-carbonyl precursors and Ru-, Rh-, and Ir-NP/IL dispersions as biphasic liquid-liquid hydrogenation nanocatalysts for cyclohexene. Chem. Eur. J. 2010, 16, 3849-3858, S3849/3841-S3849/3834.

[86] Du JQ, Zhang Y, Tian T, Yan SC, Wang HT. Microwave irradiation assisted rapid synthesis of $\mathrm{Fe}-\mathrm{Ru}$ bimetallic nanoparticles and their catalytic properties in water-gas shift reaction. Mater. Res. Bull. 2009, 44, 1347-1351.

[87] Arquilliere PP, Santini CC, Haumesser PH, Aouine M. Synthesis of copper and copper-ruthenium nanoparticles in ionic liquids for the metallization of advanced interconnect structures. ECS Trans. 2011, 35, 11-16.

[88] Helgadottir IS, Arquillière PP, Campbell PS, Santini CC, Haumesser PH. Novel chemical route to size-controlled $\mathrm{Ta}(0)$ and Ru-Ta Nanoparticles in ionic liquids. MRS Online Proc. Library 2012, 1473.

[89] Shah A, Latif-ur-Rahman, Qureshi R, Zia-ur-Rehman. Synthesis, characterization and applications of bimetallic (Au-Ag, Au-Pt, Au-Ru) alloy nanoparticles. Rev. Adv. Mater. Sci. 2012, 30, 133-149.

[90] Venkatesan R, Prechtl MHG, Scholten JD, Pezzi RP, Machado G, Dupont J. Palladium nanoparticle catalysts in ionic liquids: synthesis, characterisation and selective partial hydrogenation of alkynes to Z-alkenes. J. Mater. Chem. 2011, 21, 3030-3036.

[91] Okazaki K-i, Kiyama T, Hirahara K, Tanaka N, Kuwabata S, Torimoto T. Single-step synthesis of gold-silver alloy nanoparticles in ionic liquids by a sputter deposition technique. Chem. Commun. 2008, 0, 69-693.

[92] Suzuki T, Suzuki S, Tomita Y, Okazaki K-i, Shibayama T, Kuwabata S, Torimoto T. Fabrication of transition metal oxide nanoparticles highly dispersed in ionic liquids by sputter deposition. Chem. Lett. 2010, 39, 1072-1074.

[93] Richter K, Birkner A, Mudring A-V. Stability and growth behavior of transition metal nanoparticles in ionic liquids prepared by thermal evaporation: how stable are they really?. Phys. Chem. Chem. Phys. 2011, 13, 7136-7141.

[94] von Prondzinski N, Cybinska J, Mudring A-V. Easy access to ultra long-time stable, luminescent europium(II) fluoride nanoparticles in ionic liquids. Chem. Commun. 2010, 46, 4393-4395.

[95] Richter R, Campbell PS, Baecker T, Schimitzek A, Yaprak D, Mudring AV. Phys. Status Solidi B 2013, 250, 1152-1164.

[96] Banerjee A, Theron R, Scott RWJ. Highly Stable noble-metal nanoparticles in tetraalkylphosphonium ionic liquids for in situ catalysis. ChemSusChem 2012, 5, 109-116. 


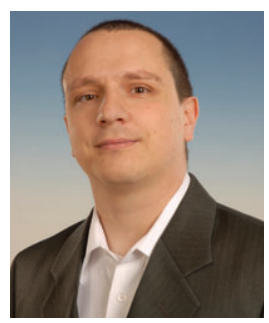

Martin H.G. Prechtl studied chemistry and food chemistry (19992004) at the University of Wuppertal (Germany) and at the University of São Paulo (Brazil). He performed research in homogeneous catalysis at the Max Planck Institute for Coal Research (Germany) and obtained his PhD from RWTH Aachen in 2007 under the supervision of Walter Leitner and David Milstein (Weizmann Institute, Israel). As Feodor Lynen fellow of the Alexander von Humboldt Foundation, he performed research about "nanocatalysis in ionic liquids" with Jairton Dupont at the Federal University of Rio Grande do Sul (UFRGS) in Porto Alegre (Brazil) and with Thomas Braun and Erhard Kemnitz at the Humboldt University Berlin (Germany) from 2007 to 2010 in the field of nanoscale catalysts in multiphase systems. He received the Scientist Returnee Award 2009 (MIWFNRW) and accepted a call of the University of Cologne as an independent group leader in 2010. He coauthored 30 articles and book chapters and edited one special issue about nanocatalysis.

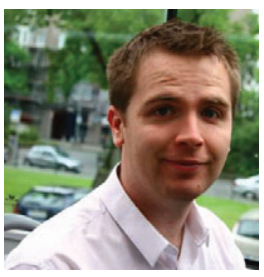

Paul S. Campbell graduated with a Master's degree in Chemistry (MChem) from Durham University, UK, in 2007. He then obtained his PhD in 2010 at the Université Claude Bernard Lyon 1, France, under the guidance of Dr. C. Santini and Nobel Laureate Yves Chauvin. There he investigated ILs as media for the separation of zirconium and hafnium as well as for metal NP synthesis and in situ catalysis. Since 2011, he has been an Alexander von Humboldt research fellow with Prof. A.-V. Mudring at the Ruhr-Universität Bochum, Germany, where his research interests include the use of ILs as a novel means to obtain advanced luminescent materials. He currently holds four patents and has coauthored 15 articles covering a broad range of aspects in IL chemistry. 